\title{
SLC5A3-dependent myo-inositol auxotrophy in acute myeloid leukemia
}

Yiliang Wei ${ }^{1}$, Yu-Han Huang ${ }^{1}$, Damianos S. Skopelitis ${ }^{1}$, Shruti V. Iyer ${ }^{1,2}$, Ana S. H. Costa ${ }^{1}$, Zhaolin Yang $^{1}$, Melissa Kramer ${ }^{1}$, Emmalee R. Adelman ${ }^{3}$, Olaf Klingbeil ${ }^{1}$, Osama E. Demerdash ${ }^{1}$, Sofya A. Polyanskaya $^{1,4}$, Kenneth Chang ${ }^{1}$, Sara Goodwin ${ }^{1}$, Emily Hodges ${ }^{5}$, W. Richard McCombie ${ }^{1}$, Maria E. Figueroa $^{3}$, and Christopher R. Vakoc ${ }^{1,6^{*}}$

${ }^{1}$ Cold Spring Harbor Laboratory, Cold Spring Harbor, NY 11724, USA

${ }^{2}$ Stony Brook University, Stony Brook, NY 11794, USA

${ }^{3}$ Sylvester Comprehensive Cancer Center, Department of Human Genetics, University of Miami, Miller School of Medicine, Miami, FL 33136, USA

${ }^{4}$ School of Biological Sciences, Cold Spring Harbor Laboratory, Cold Spring Harbor, NY 11724, USA ${ }^{5}$ Department of Biochemistry and Vanderbilt Genetics Institute, Vanderbilt University School of Medicine, Nashville, TN 37232, USA

${ }^{6}$ Lead contact

*Correspondence: vakoc@ cshl.edu; Christopher R. Vakoc, 1 Bungtown Rd, Cold Spring Harbor, NY 11724. 516-367-5030

Running title: myo-inositol auxotrophy in AML Category: Myeloid Neoplasia

\section{CONFLICT OF INTEREST DISCLOSURES}

C.R.V. has received consulting fees from Third Rock Ventures, Roivant Sciences, and C4 Therapeutics, has served on the scientific advisory board of KSQ Therapeutics and Syros Pharmaceuticals, and has received research funding from Boehringer-Ingelheim during the conduct of the study.

\section{FINANCIAL SUPPORT}

This work was supported by Cold Spring Harbor Laboratory NCI Cancer Center Support grant 5P30CA045508. Additional funding was provided to C.R.V. by the Pershing Square Sohn Cancer Research Alliance, National Institutes of Health grants R01 CA174793 and P01 CA013106, a Leukemia \& Lymphoma Society Scholar Award. M.EF. is partially supported by a Scholar Award 
from the Leukemia \& Lymphoma Society and by a Leukemia \& Lymphoma Society SCOR award (7071-18). E.R.A. was supported by the T32-CA217835 Cancer Epigenetics Training grant to the University of Miami.

\begin{abstract}
An enhanced requirement for nutrients is a hallmark property of cancer cells. Here, we optimized an in vivo genetic screening strategy in acute myeloid leukemia (AML), which led to the identification of the myo-inositol transporter SLC5A3 as a dependency in this disease. We demonstrate that SLC5A3 is essential to support a myo-inositol auxotrophy in AML. The commonality among SLC5A3-dependent AML lines is the transcriptional silencing of ISYNA1, which encodes the rate limiting enzyme for myoinositol biosynthesis, inositol-3-phosphate synthase 1. We use gain- and loss-of-function experiments to reveal a synthetic lethal genetic interaction between ISYNAI and SLC5A3 in AML, which function redundantly to sustain intracellular myo-inositol. Transcriptional silencing and DNA hypermethylation of ISYNAI occur in a recurrent manner in human AML patient samples, in association with $I D H 1 / I D H 2$ and $C E B P A$ mutations. Our findings reveal myo-inositol as a nutrient dependency in AML caused by the aberrant silencing of a biosynthetic enzyme.
\end{abstract}




\section{Statement of significance}

We show how epigenetic silencing can provoke a nutrient dependency in AML by exploiting a synthetic lethality relationship between biosynthesis and transport of myo-inositol. Blocking the function of this solute carrier may have therapeutic potential in an epigenetically-defined subset of AML. 


\section{Introduction}

A consistent property of cancer cells is metabolic dysfunction, which can occur to support the energetic and biosynthetic requirements of a rapidly growing tumor (1). One manifestation of this process is that tumor cells become more dependent on extracellular nutrient uptake for their growth and viability than normal tissues, a process referred to as auxotrophy (2). In many tumors, auxotrophies are driven by an elevated anabolic demand for metabolite building blocks, as is the case for serine (3), glutamine (4), and cysteine (5). However, cancer cells can also develop nutrient dependencies through an acquired defect in de novo metabolite biosynthesis pathways. One of the clearest examples of this is the dependence of lymphoid cancers on extracellular asparagine, which occurs because the rate limiting enzyme in asparagine biosynthesis becomes epigenetically silenced in this malignancy (6). This nutrient dependency can be exploited by infusion of recombinant asparaginase, which is an approved chemotherapeutic in acute lymphoblastic leukemia that depletes asparagine from the plasma (7). Arginine and cholesterol auxotrophies in cancer have also been attributed to diminished expression of key biosynthetic enzymes $(8,9)$. While these examples point to the therapeutic significance of auxotrophy in human cancer, few systematic surveys of nutrient dependencies have been performed in cancer to date.

Solute carriers (SLCs) are an important class of transporter proteins that regulate nutrient utilization in normal and neoplastic contexts. The human genome encodes over 400 SLC proteins organized into 65 subfamilies, which regulate intracellular levels of amino acids, sugars, ions, lipids, neurotransmitters, and drugs (10). SLCs contain transmembrane alpha helices that form a substrate binding channel, which often use ion gradients to co-transport substrates into cells (11). Owing to their specificity for transporting specific substrates, genetic targeting of SLCs provides a powerful tool for manipulation of intracellular metabolite levels and for exploration of nutrient dependencies $(12,13)$. Numerous SLC genes are mutated in human genetic disorders and an emerging body of evidence suggests a role for 
SLC proteins in the pathogenesis of human cancer $(14,15,16)$. Moreover, the substrate binding cavity of SLC proteins can be blocked with small molecules, which has fueled an interest in this class of proteins as drug targets $(16,17,18)$.

Myo-inositol is an abundant carbocyclic sugar alcohol in eukaryotic cells $(19,20)$. One of the most well-characterized functions of myo-inositol is as a head group component of lipid phosphatidylinositols (PIs), which are important signaling molecules that function downstream of growth factor signaling. In this lipid context, the phosphorylation state of myo-inositol is highly regulated by kinases (e.g. PI3K) and phosphatases (e.g. PTEN) to control the AKT-mTOR signaling axis (21). In addition, phospholipase $\mathrm{C}$ can release inositol triphosphates from PIs, which function as a second messenger that promotes the intracellular release of calcium from the endoplasmic reticulum $(22,23)$. Myo-inositol is also an osmolyte, whose intracellular concentrations can be regulated to allow cells to survive in hypertonic environments $(24,25)$.

Intracellular myo-inositol can be derived from three different sources: (1) de novo biosynthesis from glucose $(26,27)$; (2) regeneration from inositol phosphates via the action of phosphatases (28); or (3) uptake from the extracellular environment by inositol transporters (29). For de novo biosynthesis, glucose is first phosphorylated by hexokinase and then converted to myo-inositol-1-phosphate by the enzyme inositol-3-phosphate synthase 1 (ISYNA1), and finally dephosphorylated by inositol monophosphatase to form free myo-inositol. Importantly, ISYNA1 catalyzes the rate-limiting step in myo-inositol biosynthesis (30). For extracellular uptake, there are three known myo-inositol SLC transporters in human, SLC5A3 (31), SLC5A11 (32), and SLC2A13 (33). Both SLC5A3 and SLC5A11 are sodium ion coupled inositol transporters, whereas SLC2A13 is a proton-coupled inositol transporter (29). Among these transporters, SLC5A3 is the most widely expressed across different tissues and has the highest affinity for myo-inositol $(33,34,35,36,37)$. 
In this study, we set out to identify genetic dependencies needed for the growth of acute myeloid leukemia cells in vivo. This approach identified the myo-inositol transporter SLC5A3 as an in vivorelevant dependency unique to AML. We show that this dependency is caused by aberrant silencing of ISYNA1. While loss of ISYNA1 has no detectable effect on the fitness of AML cells, it causes an enhanced dependency on SLC5A3 and on extracellular myo-inositol for cell viability. Taken together, our findings reveal myo-inositol auxotrophy as metabolic vulnerability in AML. 


\section{Results}

\section{A domain-focused CRISPR screening method for identifying in vivo-relevant AML dependencies}

The genetic dependencies of a cancer cell can be markedly altered by in vitro versus in vivo growth conditions (38), particularly the requirement for cell surface receptors and metabolic enzymes whose function is influenced by the extracellular microenvironment. This issue motivated us to develop a robust screening method for interrogating genetic dependencies of human AML cells grown in vivo. For this study, we employed our previously cloned domain-focused sgRNA libraries targeting transcription factors (39), chromatin regulators (40, 41), and kinases (42). In addition, we cloned domain-focused sgRNA libraries targeting G-protein coupled receptors, ion channels, solute carriers, glycosyltransferases, proteases, and membrane proteins, which were all cloned into the LRG2.1T vector backbone which contains an optimized sgRNA2.1 scaffold (43). Our screens were performed by lentiviral transduction of each sgRNA library into the AML cell line MOLM-13, engineered to express Cas9 and firefly luciferase. A key optimization step was to select for high engraftment efficiency of this MOLM-13 line through pre-transplantation before running the pooled screen (Figure 1A). After transduction with an sgRNA library, MOLM-13 cells were injected via tail vein into 10-20 immunedeficient mice. MOLM-13 cells were allowed to expand for 12 days in vivo, followed by the mice being sacrificed and genomic DNA being isolated from bone marrow and spleen tissue for deep sequencing of the PCR-amplified sgRNA cassette (Figure 1A). While establishing this procedure, we found that pooling of bone marrow or spleen samples prior to PCR maximized sgRNA representation and the overall accuracy of the screens. Importantly, the sgRNA read count distribution (a reflection of screen quality) and the depletion pattern of control sgRNAs of in vivo samples was comparable to the same screen being performed in vitro (Figure S1, Supplementary Table 1). We have previously characterized AML dependencies in vitro using sgRNA libraries targeting kinases (42), transcription factors (39), and chromatin regulators (40, 41). The in vivo CRISPR screening showed highly consistent results with our previous findings (Figure 1B-D). In addition, the in vivo screen results 
between pooled spleen and bone marrow samples were consistent as well (Figure S2). The overall accuracy of our screen was further supported by the in vivo-specific depletion of sgRNAs targeting CD47 $(44,45,46)$ and CXCR4 (47), which are known AML dependencies that regulate interactions with the in vivo microenvironment (Figure 1E). In contrast to previous in vivo RNAi screening in leukemia that shows only $\sim 10 \%$ of in vivo dependencies overlapping with in vitro targets (48), our screening showed largely consistent results between in vivo and in vitro conditions (Figure 1F, Figure S3, and Supplementary Table 1). From this screening effort targeting a total of 5,768 genes, we identified 239 targets as in vivo-relevant dependencies (Figure 1F, Supplementary Table 1).

\section{SLC5A3 dependency is in vivo-relevant and AML-specific}

While our original intent was to identify in vivo-specific dependencies, the scarcity of such hits in our screen prompted us to explore instead whether any in vivo-validated dependencies were unique to AML relative to other cancers. For this purpose, we turned to Project Achilles/DepMap, which has performed genome-wide CRISPR screens for essential genes in $>769$ diverse cancer cell lines grown in vitro $(49,50)$. Through analysis of these data, we nominated myeloid lineage TFs (MYB, CBFB, and SPI1) and the myo-inositol transporter protein SLC5A3 as the top leukemia-biased dependencies among the pool of in vivo-validated targets (Figure 2A). The AML specificity of SLC5A3 was distinct from other essential SLC genes, which tended to be needed across diverse cancer types (Figure 2B). The in vivo screening in MOLM-13 cells and our own in vitro SLC screening in 10 diverse cancer cell lines corroborated SLC5A3 as a unique AML dependency under in vitro and in vivo conditions (Figure 2C, Figure S4A-E). Unlike the aforementioned myeloid TFs, no prior study has investigated the role for SLC5A3 in AML, which motivated our subsequent evaluation of this target.

\section{Validation of SLC5A3 dependency in a subset of AML cell lines}


To validate SLC5A3 as a dependency in AML, we performed competition-based proliferation assays following lentiviral transduction with sgRNA-expressing vectors in Cas9-expressing AML cell lines. The genome editing efficiency of these sgRNAs targeting SLC5A3 was validated using genomic DNA qPCR with primers that hybridize to the Cas9 target sequence (Figure S5A). Using this approach, we validated that the cell fitness of several AML lines was impaired by SLC5A3 sgRNAs (e.g. MOLM-13, SEM, MV4-11, U937, EOL1), whereas other AML lines were SLC5A3 independent (e.g. HEL, KASUMI, SET-2) (Figure 3A, Figure S5B-C). As a control, an sgRNA targeting the DNA replication protein PCNA suppressed the growth of all cancer cell lines tested (Figure 3A, Figure S5B). cDNA rescue experiments also supported that these cell fitness alterations were due to a requirement for the SLC5A3 gene product and rule out off target effects as contributing to this phenotype (Figure S6A-E). In MOLM-13 cells, loss of SLC5A3 led to cell cycle arrest (Figure S7A-B). We also verified that knockout of SLC5A3 suppressed the growth of MOLM-13 cells under in vivo conditions (Figure 3B-E, Figure S8A-F). Collectively, these findings demonstrate that SLC5A3 is essential in a subset of AML cell lines under in vitro and in vivo conditions.

\section{SLC5A3-dependent AML lines are myo-inositol auxotrophs}

Since the primary substrate for SLC5A3 is myo-inositol (29), we performed liquid chromatography mass spectrometry (LC-MS) to measure intracellular myo-inositol levels in cancer cell lines following SLC5A3 inactivation. As expected, knockout of SLC5A3 in MOLM-13 or MV4-11 cells led to a significant reduction of intracellular myo-inositol, whereas the level of other metabolites (e.g. glutamine) was unaffected (Figure 4A). This finding prompted us to evaluate the impact of removing myo-inositol from the media of AML cell lines. Remarkably, SLC5A3-dependent cell lines MOLM-13, MV4-11, and U937 slowed their growth following myo-inositol depletion, whereas the growth of SLC5A3-independent lines HEL, SET-2, and K562 was unaffected by myo-inositol depletion (Figure 
4B-C). Taken together, these experiments suggest that SLC5A3 dependency is associated with a myoinositol auxotrophy in AML.

It has been shown that developmental abnormalities in $S l c 5 a 3^{-/-}$mice can be rescued by providing supra-physiological concentrations myo-inositol in the diet, which can be transported into cells in a SLC5A3-independent manner via low affinity transporters $(51,52,53)$. This observation motivated us to perform analogous experiments in SLC5A3-dependent AML cell lines by supplementing normal growth media with additional myo-inositol. This revealed that a $\sim 5$-fold increase in myo-inositol concentrations in regular growth media (RPMI) was sufficient to bypass the necessity of SLC5A3 in MOLM-13 and MV4-11 cell line contexts (Figure 4D). These findings lend strong support that the essential function of SLC5A3 in AML is the transport of extracellular myo-inositol into the cell interior.

\section{Transcriptional silencing of ISYNA1 leads to SLC5A3 dependency through a synthetic lethal genetic interaction}

We next investigated the mechanism underlying SLC5A3 dependency and myo-inositol auxotrophy in AML. Importantly, SLC5A3 dependency did not correlate with expression of SLC5A3, nor did it correlate with silencing of the alternative myo-inositol transporters SLC5A11 and SLC2A13 (Figure S9A-C). Furthermore, triple knockout experiments targeting SLC5A3, SLC5A11, and SLC2A13, failed to reveal evidence of redundancy (Figure S10A-B). These results led us to consider whether the myo-inositol auxotrophy in AML was due instead to an acquired defect in de novo myo-inositol biosynthesis (Figure 5A). By analyzing the mRNA levels of known myo-inositol biosynthesis genes, we noticed a significant inverse correlation between SLC5A3 dependency and expression of ISYNA1, which encodes the rate-limiting enzyme in myo-inositol biosynthesis (30) (Figure 5B). We confirmed 
the heterogeneous expression of ISYNA1 in AML cell lines using western blotting, which mirrored the heterogeneity of mRNA levels (Figure 5C, Figure S11A).

The findings above led us to hypothesize that a genetic redundancy may exist between ISYNA1 and SLC5A3, which represent two independent strategies for maintaining intracellular myo-inositol levels. To evaluate this possibility, we targeted ISYNA1 and SLC5A3 (alone and in combination) in HEL and K562, which are leukemia lines that express both genes. Competition-based proliferation assays using bi-cistronic sgRNA vectors demonstrated that dual targeting of ISYNAI and SLC5A3 led to a more severe proliferation defect than the loss of each gene individually (Figure 5D-E, Figure S11B). LC-MS analysis demonstrated that a combined deficiency of ISYNA1 and SLC5A3 also led a more severe decrease in intracellular myo-inositol than observed following either single gene knockout (Figure S11C). Knockout of ISYNA1 in HEL cells also led to a significant reduction of intracellular myoinositol when cultured in myo-inositol deficient media (Figure S11D). In addition, knockout of ISYNA1 was sufficient to convert HEL and K562 cells into myo-inositol auxotrophs (Figure 5F-G). Taken together, these experiments validate a genetic redundancy between ISYNA1 and SLC5A3 to sustain intracellular myo-inositol and cell viability.

To validate that ISYNA1 silencing is the underlying cause of myo-inositol auxotrophy and SLC5A3 dependency in AML, we lentivirally expressed a ISYNA1 cDNA in AML cell lines that lacked endogenous ISYNA1 expression (MOLM-13, MV4-11, EOL1, and U937) (Figure 6A). Remarkably, restoring ISYNA1 was sufficient to elevate myo-inositol levels and bypass the essentiality of SLC5A3 when cultured in normal growth media (Figure 6B-C, Figure S12). This finding was further corroborated using a genome-wide screening approach, which revealed SLC5A3 as the sole genetic dependency rescued by ISYNA1 cDNA expression (Figure S13A-E). Furthermore, forced ISYNA1 expression also eliminated the myo-inositol auxotrophy (Figure 6D). 
To validate that ISYNA1 is the key enzyme for de novo myo-inositol biosynthesis from glucose, we performed stable isotope tracing experiment by treating the cells with ${ }^{13} \mathrm{C}$ labeled glucose for 48 hours and measuring intracellular ${ }^{13} \mathrm{C}$ labeled myo-inositol by LC-MS (Figure S14A). In MOLM-13 cells, the labeled glucose was only converted into myo-inositol when ISYNA1 was overexpressed (Figure S14B). On the contrary, a significant reduction of intracellular ${ }^{13} \mathrm{C}$ labeled myo-inositol was observed in HEL cells following ISYNA1 knockout (Figure S14C).

\section{Aberrant ISYNA1 promoter hyper-methylation occurs recurrently in human AML patient samples}

We next evaluated whether epigenetic silencing of ISYNAI occurs in human AML patient samples. In the cell line AML models, we noticed that transcriptional silencing of ISYNAI was associated with DNA hypermethylation and the loss of histone acetylation in the vicinity of its CpG island/promoter (Figure S15A-B). Using Nanopore sequencing, we confirmed DNA hypermethylation at the ISYNAI gene in MOLM-13 and MV4-11 lines, and hypomethylation in the HEL line (Figure 7A, Figure S15C). Notably, these regions remained unmethylated in normal human hematopoietic stem and progenitor cells (HSPC) (54) (Figure 7A). Having established hypermethylation as a biomarker of ISYNA1 silencing, we next evaluated whether this genomic region was hypermethylated in human AML patient samples analyzed by The Cancer Genomic Atlas (TCGA), in which 170 genetically annotated samples were evaluated using Illumina Infinium 450k DNA methylation assay (55). In this study, we found that ISYNAI hypermethylation was present in $~ 20 \%$ of human AML samples (z score $>0.5$ ), whereas other flanking regions lacked heterogeneity in methylation (Figure 7B, Figure S15D-E). Importantly, the presence of hypermethylation correlated with lower ISYNA1 expression (Figure 7C, Figure S15F). In addition, we found that ISYNA1 hypermethylation was significantly enriched for patients that possess $I D H 1 / I D H 2$ or $C E B P A$ mutations (Figure 7B, D). The TCGA AML patient gene expression 
data further shows patients with IDH2 mutations have significantly lower ISYNA1 gene expression (Figure 7E). We further evaluated ISYNA1 methylation in an independent cohort of 119 AML patient samples analyzed using reduced representation bisulfite sequencing (56), which likewise identified hypermethylation in the ISYNA1 genic region in a subset of AML cases, which was absent in normal bone marrow cells (Figure S16). Taken together, these findings validate that aberrant hypermethylation and silencing of ISYNAI occurs recurrently in AML. 


\section{Discussion}

Our study identifies a myo-inositol auxotrophy as a previously unrecognized metabolic vulnerability in AML. We trace this auxotrophy to aberrant silencing of ISYNA1, which is the rate-limiting enzyme for myo-inositol biosynthesis. Our work suggests that normal cells maintain intra-cellular myo-inositol levels through the redundant mechanisms of de novo biosynthesis and SLC5A3-dependent transport from the extracellular microenvironment. Our work shows how ISYNAl silencing eliminates this redundancy, which in turn drives an elevated demand for SLC5A3-dependent myo-inositol transport in AML (Figure 7F).

The evolution of redundant mechanisms for sustaining intracellular myo-inositol is likely to reflect the vital function of this metabolite, which is well-supported by experiments in diverse eukaryotic species (57). Myo-inositol is incorporated as head group into a variety of lipids, which function to promote signal transduction in growth-promoting pathways (e.g. AKT-mTOR) (21). Hence, blockade of SLC5A3 in ISYNA1-deficient AML is likely to extinguish AKT-mTOR signaling, which would be expected to cause an acute arrest in cell proliferation. Targeting of SLC5A3 would also be expected to deplete the cell of inositol phosphates, which regulate calcium release as well as the function of chromatin remodeling complexes $(57,58)$. These myriad functions of myo-inositol for cell viability are likely to contribute to the fitness defects of targeting SLC5A3 in AML.

Our study adds to a growing body of evidence highlighting how epigenetic silencing can alter metabolic pathways and lead to nutrient dependencies. Hypermethylation of the argininosuccinate synthetase (ASS1) gene promoter leads to an arginine dependency in several human cancers $(8,59,60)$. Similarly, hypermethylation and silencing of asparagine synthetase (ASNS) has been linked to asparagine auxotrophy in lymphoid leukemia $(6,61,62)$. Promoter hypermethylation of a rate-limiting 
cholesterol biosynthetic enzyme, squalene mono-oxygenase (SQLE) leads to a cholesterol auxotrophy in anaplastic large cell lymphoma (9). It remains unclear as to why the $\mathrm{CpG}$ islands of metabolic genes are prone to DNA hyper-methylation, as it is unclear whether silencing of these genes confers a growth advantage in human cancer cells. Since epigenetic and metabolic regulation is tightly interconnected, it is possible that feedback regulation exists between the chromatin regulatory machinery and metabolite levels. For example, we observe that ISYNA1-silenced AML is correlated with the presence of IDH1/IDH2 gain-of-function mutations, which produce 2-hydroxyglutarate which in turn inhibits active DNA demethylation $(63,64)$. Thus, it is possible that the unique metabolic state of AML is the predisposing factor for ISYNA1 silencing as a passenger epigenetic event in this disease, a possibility that warrants additional investigation.

The aberrant DNA methylation of ISYNA1 is unlikely to be unique to AML, as several other cancer types (e.g. breast invasion carcinoma) we surveyed in TCGA database also showed a similar pattern (Figure S17A-F). However, the correlation between SLC5A3 dependency and ISYNA1 expression is weak in non-AML cancer cell lines. This suggest that other factors might influence myo-inositol levels in non-AML contexts. Nevertheless, these findings raise the possibility that the SLC5A3 dependency and MI auxotrophy might be present more broadly across human cancers.

Several mechanisms have been described by which the ISYNAI gene is regulated in mammalian cells to control myo-inositol biosynthesis, which may predispose to gene silencing in AML. For example, the inositol hexakisphosphate kinase 1 (IP6K1) has been found in the nucleus where it acts directly to maintain transcription of ISYNAI and prevent DNA hypermethylation (65). In addition, prior studies have shown that Musashi2, a known oncogene, regulates ISYNA1 expression in the context of cancer (66). The tumor suppressor p53 has also been shown to directly regulate the ISYNAI promoter (67). 
Our own genetic analysis of human samples suggests that mutations of the C/EBP $\alpha$ transcription factor are associated with ISYNAI hyper-methylation, which may influence the methylation state of this gene. Collectively, these studies suggest that several cancer-relevant pathways converge in regulating ISYNA1 expression, which might underlie the aberrant silencing of this gene in AML.

Our work raises the possibility that the acquired dependency on SLC5A3 and extra-cellular myoinositol in AML might have therapeutic significance. In analogy to the use of asparaginase in lymphoblastic leukemias (68), one possibility for therapeutic intervention in ISYNA1-deficient AML would be to suppress plasma levels of myo-inositol using an infusion of catabolic enzymes. MIOX (myo-inositol oxygenase) is one such enzyme that oxidizes myo-inositol into D-glucuronic acid (69, 70). Another enzyme capable of catabolizing myo-inositol is inositol dehydrogenase from Bacillus subsilis $(71,72)$. As an alternative strategy, several solute carriers have been targeted using small molecules (15). Thus, an attractive strategy would be to suppress SLC5A3 function. While Slc5a3-null mice die soon after birth due to respiratory failure from abnormal development of peripheral nerves, this phenotype can be rescued by prenatal myo-inositol supplementation. Adult Slc5a3-null mice show a decrease of tissue myo-inositol levels and reduced nerve conduction velocity, which can also be alleviated by myo-inositol supplementation (52). Taken together, our findings justify an investigation of myo-inositol lowering agents or SLC5A3 blockade as therapeutic interventions for the elimination of ISYNA1-deficient AML cells. 


\section{Material and Method}

\section{Cell Lines and Culture}

All cell lines were authenticated using STR profiling. MOLM-13, MV-4-11, SEM, OCI-AML3, EOL1, U937, NOMO-1, HEL, SET-2, KASUMI-1 (human acute myeloid leukemia, AML), K562 (human chronic myeloid leukemia, CML), AsPC-1, MIA PaCa-2 (human pancreatic cancer) and NCI-H82 (human small cell lung cancer, SCLC) cells were cultured in RPMI supplemented with 10\% fetal bovine serum (FBS). MA9-ITD, MA9-RAS (engineered human AML) cells were cultured in IMDM supplemented with 20\% FBS $(73,74)$. RH30, RD (human rhabdomyosarcoma), and HEK293T cells were cultured in DMEM supplemented with 10\% FBS. NCI-H1048 (human SCLC) was cultured in DMEM:F12 supplemented with $0.005 \mathrm{mg} / \mathrm{mL}$ insulin, $0.01 \mathrm{mg} / \mathrm{mL}$ transferrin, $30 \mathrm{nM}$ sodium selenite, $10 \mathrm{nM}$ hydrocortisone, $10 \mathrm{nM} \beta$-estradiol, $4.5 \mathrm{mM}$ L-glutamine, and 5\% FBS. Penicillin/streptomycin was added to all cell culture. All cell lines were cultured at $37^{\circ} \mathrm{C}$ with $5 \% \mathrm{CO}_{2}$, and were periodically tested negative for mycoplasma contamination. All experiments were performed within one month of thawing a cryopreserved vial of cells.

To make myo-inositol deficient cell culture (-MI), customized RPMI medium without myo-inositol (Bio-Techne) was supplemented with dialyzed FBS (\#26400044, ThermoFisher Scientific) and penicillin/streptomycin. The dialyzed FBS shows 100 times less myo-inositol level compared to regular FBS, validated by LC-MS. To measure cell growth in (-MI) cell medium as shown in Figure 4, cells were washed twice with PBS to remove residue cell medium, and then resuspended in (-MI) medium with one million cells per $\mathrm{mL}$. One $\mathrm{mL}$ cells were transferred into one well in 12 -well plates, with three replicates for each condition. Cell number and viability was measured using automated cell counter (Invitrogen Countess) every two days. For each passage, $0.4 \times 10^{6}$ (for MOLM-13 cells) or $0.2 \times 10^{6}$ (for other lines) cells were passed on to the next passage. For MOLM-13, MV4-11, and U937 
lines that are sensitive to myo-inositol depletion, the cell numbers dropped drastically after $2^{\text {nd }}$ or $3^{\text {rd }}$ passage in MI-deficient medium. For these samples, 1/5 volume of the cells were passed to the next passage. We performed at least 2 biological replicates (each included 3 technical replicates) for each cell line tested. For (+MI) cell medium, $0.2 \mathrm{~g} / \mathrm{L}(1.11 \mathrm{mM})$ myo-inositol (\#I5125, Sigma) was added into the (-MI) medium.

\section{Animals}

NOD.Cg-Prkdc ${ }^{\text {scid }} I l 2 r g^{t m 1 W j l} \operatorname{Tg}(\mathrm{CMV}-\mathrm{IL} 3, \mathrm{CSF} 2, \mathrm{KITLG}) 1 \mathrm{Eav} / \mathrm{MloySzJ}$ (known as NSGS) mice expressing human IL3, GM-CSF, and SCF were used for in vivo CRISPR screening. NOD.Cg-

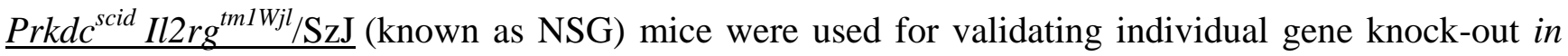
vivo. All animals were purchased from The Jackson Laboratory (jax.org). Animal procedures and studies were reviewed and approved by the Institutional Animal Care and Use Committee (IACUC) at Cold Spring Harbor Laboratory.

\section{Plasmid Construction}

The lentiviral Cas9 vector (LentiV_Cas9_puro/Addgene \#108100), the lentiviral sgRNA vectors (LRG2.1-GFP/Addgene \#108098, LRG2.1-mCherry/Addgene \#108099, the lentiviral luciferase vector (LentiV_Neo_Luc/Addgene \#105621), and the lentiviral cDNA expression vector (LentiV_Neo/Addgene \#108101) have been described in previous studies $(42,75)$. DNA oligos of the sgRNAs were cloned into LRG2.1-GFP/mCherry vectors using a BsmBI restriction site. The cDNAs of ISYNA1 (\#OHu18552, GenScript) and SLC5A3 (\#53836, Addgene) were cloned into LentiV_Neo vector using In-Fusion cloning system (Takara Bio), and flag tag was inserted to the C-terminus of the cDNAs. The same system was used to mutate the PAM sequences in the SLC5A3 gene. Dual targeting vectors were generated by inserting sgRNA1-scaffold-bU6-sgRNA2 sequence (synthesized as geneblocks by IDT) into the BsmBI site of LRG2.1-GFP-P2A-BlastR vector using Gibson Assembly 
(NEB). The scaffold sequence for sgRNA1 was generated by replacing the stem-stem loop region of the LRG2.1 scaffold (76) with a previously described CRISPRi sgRNA scaffold (77).

\section{Construction of Domain-Focused sgRNA Library}

The Kinase sgRNA library and Transcription factor sgRNA library were described in the previous studies $(39,42)$. The other sgRNA libraries were generated in a similar fashion. The gene lists were obtained from HUGO Gene Nomenclature Committee database (HGNC, genenames.org). The domainfocused sgRNA libraries were designed by using CSHL CRISPR sgRNA design tool (crispr.cshl.edu), and at least 6 sgRNAs were selected for each gene or domain. Non-targeting sgRNAs as negative control, and sgRNAs targeting essential genes as positive control, were added into the pooled sgRNA library. The pooled library was synthesized on an array platform (Twist Bioscience) and then cloned into the BsmBI restriction site of LRG2.1-GFP vector using Gibson Assembly System (NEB).

\section{Lentivirus Transduction}

Lentivirus was produced in HEK293T cells as described in the previous study (42). Briefly, $10 \mathrm{ug}$ of plasmid DNA was mixed with helper plasmids (5 ug VSVG and $7.5 \mathrm{ug} \mathrm{psPAX} 2$ ) and $1 \mathrm{mg} / \mathrm{mL}$ polyethylenimine (PEI 25000). The mix was then used to transfect HEK293T cells in $10 \mathrm{~cm}$ dish. The mix was replaced by fresh cell media 6 hours post the transfection, and lentivirus-containing supernatant was collected at 24, 48, and 72 hours post-transfection. To infect cells with lentivirus, the target cells were mixed with the virus and $8 \mathrm{ug} / \mathrm{mL}$ polybrene in 12 well plates, and then centrifuged at $1700 \mathrm{rpm}$ for 45 60 min. Cell media was changed 24 hours post infection. For cells infected with LentiV_Neo or LentiV_Blast vector, G418 or Blasticidin was added to the cell culture at day 3 post infection, respectively.

\section{Pooled in vivo CRISPR Screening}


Cas9 and Luciferase (Luc) expressing MOLM-13 cell line (MOLM-13-Cas9-Luc) was established by infecting MOLM-13 cells with LentiV_Cas9_puro and LentiV_Neo_Luc, and then selected with puromycin and G418. To improve in vivo engraftment efficiency, 5 million MOLM-13-Cas9-Luc cells were transplanted into two sub-lethally irradiated (2.5 Gy), 8-week-old female NSG mice through tail vein injection. The cells were collected from the bone marrow of the injected mice ten days post injection by flushing the tibia with phosphate buffered saline (PBS). The collected cells were then cultured in RPMI supplemented with 10\% FBS, puromycin and G418 to remove murine cells.

To determine virus titer for the sgRNA library, MOLM-13-Cas9-Luc cells were infected with different volumes of the virus using spin infection technique as described above. Flow cytometer (Guava easyCyte, Millipore) was used to determine the infection rate by measuring the percentage of GFP positive cells. The amount of virus that gave $\sim 30 \%$ infection rate was used for further infection experiments.

To ensure at least 1000 fold coverage of each sgRNA in the library, the amount of cells to be infected was calculated using the equation: cell number $=\operatorname{sgRNA}$ number/infection rate*1000. For instance, given the sgRNA number in the SLC sgRNA library was $\sim 3000$, and the infection rate was $\sim 30 \%$, at least 10 million cells were used for one CRISPR screening. Ten mice were planned to be injected, thus 100 million cells were used for the initial infection.

To perform in vivo CRISPR screening, the pre-engrafted MOLM-13-Cas9-Luc cells were infected with the library lentivirus. Three days post infection, the infection rate was checked by flow cytometer to ensure $\sim 30 \%$ GFP positive cells. For the SLC sgRNA library, the infected wells were split into three groups. Group 1, ten million cells were spin-collected and kept frozen as P0. Group 2, ten million cells were kept growing in cell culture as in vitro CRISPR screening sample, in parallel of in vivo CRISPR 
screening sample. During each passage, the percentage of GFP positive cells was measured, and the cell number was calculated using the equation above to ensure at least 1000 fold coverage of the sgRNA library. Group 3, one hundred million cells were collected, washed with PBS to remove any residual cell media, and then resuspended in $2 \mathrm{~mL}$ PBS $\left(5 \times 10^{7}\right.$ cells $\left./ \mathrm{mL}\right) .200 \mathrm{uL}$ of the cells were transplanted to one sub-lethally irradiated (2.5 Gy), 8-week-old female NSGS mouse via tail vein injection. A total number of nine mice were successfully injected. The engraftment and growth of the injected human AML cells was monitored by biofluorescence imaging (IVIS Spectrum System, Caliper Life Sciences). Images were taken 10 minutes after intraperitoneal injection of D-luciferin (50 $\mathrm{mg} / \mathrm{kg}$ ), every 3 days post injection. On day 12 post injection, the animals were euthanized. The spleen (SP) and bone marrow (BM) samples were collected. The spleen was broken down by gently crushing between the frost sides of two glass slides, and then filtered through a cell strainer. The bone marrow was flushed from tibia by injecting with PBS. Meanwhile, the in vitro samples were also spin-collected. The genomic DNA from P0, in vitro sample, individual SP and BM were purified using QIAamp DNA Mini kit (QIAGEN). The genomic DNA were then diluted into 100 ng/uL. An equal aliquot of genomic DNA from individual SP and BM were pooled to make pooled SP and pooled BM samples. The in vivo screening for other sgRNA libraries were performed in a similar fashion, with the amount of cells and the number of animals adjusted according to the sgRNA library size to ensure at least 1000X coverage.

Barcoded sequencing libraries for P0, in vitro, 9 individual SP, 9 individual BM, pooled SP, and pooled BM were prepared using the same method as described in the previous study (42). The libraries were pooled and analyzed by paired-end sequencing using Miseq (Illumina) with MiSeq Reagent Kit v3 (Illumina). 
The sequencing data was de-barcoded and subsequently mapped to the reference sgRNA library using a customized tool as described in the previous study (76). The screening data was analyzed using MAGeCK-MLE $(78,79)$. A beta score was calculated for each gene and negative control by the MAGeCK-MLE model. It is a measurement of gene selections similar to 'log fold change' in differential expression analysis (79). Negative beta score indicated negative selection, and positive beta score indicated positive selection. To calculate beta score for the non-targeting negative controls, every 6 negative controls were pooled as a single target for the MAGeCK-MLE calculation. The data was visualized using Prism 8 program.

\section{Pooled in vitro CRISPR Screening}

Ten cell lines representing five different type of cancers were used for in vitro CRISPR screening of the SLC sgRNA library, including MOLM-13, HEL, NOMO-1 (AML), K562 (CML), AsPC-1, MIA PaCa-2 (pancreatic cancer), NCI-H82, NCI-H1048 (SCLC), RH30, RD (rhabdomyosarcoma). The Cas9 expressing cell lines were established by infecting with LentiV_Cas9_puro, and then selected with puromycin. The virus titer for each cell line was determined individually as described above. To ensure 1000 fold coverage of the library, at least 10 million cells were initially infected with the library with $\sim 30 \%$ infection rate. Day 3 post infection, 10 million cells were collected and kept frozen as P0. Cells were passed every three days, cell number and GFP\% were checked every passage. Cells collected at passage 5 and 8 were saved as P5 and P8 samples. Genomic DNA extraction, sequencing library preparation, and data analysis were performed the same way as described above.

For the genome-wide CRISPR screens in Figure S13, Cas9 expressing MOLM13 and MOLM13 ${ }^{\text {ISYNA1 }}$ cells were transduced with Human CRISPR Knockout Pooled Library (Brunello, Addgene \#73178) with low MOI ( 0.3). The original library vector contains puromycin selection marker, which was replaced with blasticidin-GFP selection marker. The transduced cells were selected with blasticidin, 
and saved as P0. The cells were passaged for another 10 doublings, and saved as P10. Two biological replicates were performed. Sequencing of the sgRNAs was performed on NextSeq platform. Data analysis was performed the same way as described above.

\section{Competition-Based Cell Proliferation Assay}

Cas9 expressing cells were infected with sgRNA cloned into LRG2.1-GFP/mCherry, or dual-targeting vector. The percentage of GFP or mCherry positive cells was first measured at day 3 post infection (P0) by flow cytometer (Guava easyCyte, Millipore), and then measured every 2 3 days at each passage, till passage 5 or 6 . The relative fluorescent $\%$ for each passage was calculated using the equation: relative fluorescent $\%=[$ fluorescent $\%] /[\mathrm{P} 0$ fluorescent $\%] * 100 \%$. Decrease of the fluorescent $\%$ indicated the infected (gene knock-out) cells were out-competed by the uninfected (wide type) cells.

\section{Cell cycle arrest analysis}

Cell cycle analysis as shown in Figure S7 was performed according to the manufacturer's protocol (BD, FITC BrdU Flow Kit; Cat. No. 559619). Cells were pulsed with BrdU for 1 hour at $37{ }^{\circ} \mathrm{C}$, and then were co-stained with 4',6- diamidino-2-phenylindole (DAPI) for DNA content measurement. Cells were finally analyzed with a BD LSRFortessa flow cytometer (BD Biosciences) and FlowJo software (TreeStar). The experiments were performed in duplicates.

\section{Western Blot}

To detect the ISYNA1 expression levels in human AML cell lines, ten million cells were collected and cell pellet was resuspended in $500 \mathrm{uL} 1 \mathrm{X}$ Laemli sample buffer (Bio-Rad). The cell lysate was then boiled for $10 \mathrm{~min}$, separated by SDS-PAGE, followed by transfer to nitrocellulose membrane and immunoblotting with 1:500 diluted mouse monoclonal anti-ISYAN1(C-9) antibody (\#sc-271830, Santa Cruz Biotechnology), and then with 1:10000 diluted polyclonal rabbit anti-mouse HRP antibody 
(\#P0260, Agilent Dako). For control, mouse monoclonal anti-HSC70 (B-6) antibody (\#sc-7298, Santa Cruz Biotechnology) was used with 1:10000 dilution.

To detect the recombinant ISYAN1-flag, ten million cells were collected two weeks post infection with cDNA vectors followed by G418 selection. Protein sample preparation and immunoblotting was the same as described above. For flag-tagged proteins, 1:5000 diluted mouse monoclonal anti-flag antibody (Sigma) was used, followed by a secondary antibody of rabbit anti-mouse HRP antibody (\#P0260, Agilent Dako) with 1:10000 dilution.

\section{In vivo validation experiments}

For the in vivo validation as shown in Figure 3, pre-engraft MOLM-13-Cas9-Luc cells were infected with LRG2.1-sgROSA-GFP-BlastR or LRG2.1-sgSLC5A3-2-GFP-BlastR. At day 2 post infection, blasticidin was added to the cell culture to select for infected cells. After 3 days selection, the cells were collected, washed with PBS to remove residual media, and resuspended to $1 \mathrm{X} 10^{6}$ cells $/ \mathrm{mL}$. 100 $\mathrm{uL}$ cells were transplanted to one sub-lethally irradiated (2.5 Gy), 8-week-old female NSG mouse via tail vein injection. Five mice were injected for each sgRNA. AML development was monitored by biofluorescence imaging (IVIS Spectrum System, Caliper Life Sciences). Images were taken 10 minutes after intraperitoneal injection of D-luciferin $(50 \mathrm{mg} / \mathrm{kg})$, on day 13, 16 and 19 post injection.

For the in vivo validation as shown in Figure S8, pre-engraft MOLM-13-Cas9-Luc cells were infected with LRG2.1-sgROSA-GFP， LRG2.1-sgSLC5A3-1-GFP， LRG2.1-sgSLC5A3-2-GFP， LRG2.1sgCD47-1-GFP, or LRG2.1-sgCD47-2-GFP. Excessive lentivirus was used to ensure >90\% infection rate. The cells were then transplanted to NSG mice as described above. 
For the LC-MS analysis shown in Figure 4, 0.5 million cells were infected with LRG2.1-sgROSAGFP-BlastR or LRG2.1-sgSLC5A3-2-GFP-BlastR in 12 well plates with 6 replicates. For the LC-MS analysis shown in Figure 6, MOLM13-Cas9 cells expressing EV or ISYNA1 were infected with LRG2.1-sgROSA-GFP-BlastR or LRG2.1-sgSLC5A3-2-GFP-BlastR in 12 well plates with 5 replicates. The infected cells were selected with blasticidin at day 2 post infection, for 3 days. For the LC-MS analysis shown in Figure S11-C, HEL-Cas9 and K562-Cas9 cells were infected with indicated single or dual targeting sgRNA vectors with over $90 \%$ infection rate, in 12 well plates with 5 replicates, for 5 days. The cells were then collected for sample preparation. For the LC-MS analysis shown in Figure S11-D, HEL-Cas9 cells were infected with LRG2.1-sgROSA-GFP-BlastR or LRG2.1sgISYNA1-GFP-BlastR in 12 well plates with 5 replicates. The infected cells were selected with blasticidin at day 2 post infection for 7 days. At least $95 \%$ of GFP positive rate was achieved. The cells were then washed twice with PBS, and cultured in myo-inositol deficient medium (-MI) for another 4 days before preparation for LC-MS.

To prepare cells for LC-MS, cells were quickly washed in PBS before adding $1 \mathrm{~mL}$ of ice-cold extraction solution $\left(50 \%\right.$ methanol, $30 \%$ acetonitrile, $\left.20 \% \mathrm{H}_{2} \mathrm{O}\right)$ per four million cells. The cells were then scrapped and the suspension was snap frozen in liquid nitrogen. Samples were agitated using a Thermomixer (Eppendorf) at $1,400 \mathrm{rpm}, 4^{\circ} \mathrm{C}$ for $15 \mathrm{~min}$ followed by incubation at $-80^{\circ} \mathrm{C}$ for $1 \mathrm{~h}$. Samples were then centrifuged at $15,000 \mathrm{rpm}, 4^{\circ} \mathrm{C}$ for $10 \mathrm{~min}$. The supernatants were further centrifuged for another $10 \mathrm{~min}$. The supernatants were stored in autosampler vials at $-80^{\circ} \mathrm{C}$ until analysis.

Intracellular extracts from 5 6 independent cell cultures were analyzed for each condition. Samples were randomized in order to avoid bias due to machine drift and processed blindly. LC-MS analysis was performed using a Vanquish Horizon UHPLC system couple to a Q Exactive HF mass 
spectrometer (both Thermo Fisher Scientific). Sample extracts $(5 \mu \mathrm{L})$ were injected onto a Sequant ZIC-pHILC column $(150 \mathrm{~mm} \times 2.1 \mathrm{~mm}, 5 \mu \mathrm{m})$ and guard column $(20 \mathrm{~mm} \times 2.1 \mathrm{~mm}, 5 \mu \mathrm{m})$ from Merck Millipore kept at $45^{\circ} \mathrm{C}$. The mobile phase was composed of $20 \mathrm{mM}$ ammonium carbonate with $0.1 \%$ ammonium hydroxide in water (solvent $\mathrm{A}$ ), and acetonitrile (solvent $\mathrm{B}$ ). The flow rate was set at $200 \mu \mathrm{l} / \mathrm{min}$ with the previously described gradient (80). The mass spectrometer was operated in full MS and polarity switching mode. The acquired spectra were analyzed using XCalibur Qual Browser and XCalibur Quan Browser software (Thermo Fisher Scientific) by referencing to an internal library of compounds.

\section{Stable Isotope Tracing by LC-MS}

For tracing de novo myo-inositol synthesis from ${ }^{13} \mathrm{C}$ labeled glucose as shown in Figure $\mathrm{S} 14$, ${ }^{13} \mathrm{C}$ labeling medium was prepared by adding ${ }^{13} \mathrm{C}$ labeled glucose (Sigma-Aldrich 389374) (final concentration $2000 \mathrm{mg} / \mathrm{L}$ ) and dialyzed FBS (final concentration 10\%) to glucose free RPMI (Thermo Fisher Scientific 11879020). Cells were washed with PBS twice and seeded in 6-well plates with ${ }^{13} \mathrm{C}$ labeling medium for 48 hours. Extracts from six independent cell cultures were analyzed for each condition. Briefly, after quick removal of the culture medium, cells were washed in PBS before adding extraction solution (50\% methanol, $30 \%$ acetonitrile, $20 \% \mathrm{H}_{2} \mathrm{O}$ ). After centrifugation to remove the precipitated proteins and insoluble debris, the supernatants were collected and stored in autosampler vials at $-80^{\circ} \mathrm{C}$ until analysis. Samples were randomized to avoid bias due to machine drift, and processed blindly. LC-MS analysis was performed using a Vanquish Horizon UHPLC system coupled to a Q Exactive HF mass spectrometer (both Thermo Fisher Scientific). Sample extracts were analyzed as previously described (80). The acquired spectra were analyzed using XCalibur Qual Browser and XCalibur Quan Browser software (Thermo Fisher Scientific) by referencing to an internal library of compounds. Metabolite peak areas were corrected for natural ${ }^{13} \mathrm{C}$ abundance using the $\mathrm{R}$ package AccuCor (81). To calculate isotopologue distribution, corrected peak areas of each metabolite's 
isotopologues were normalized to the total metabolite pool (sum of all isotopologues of a given metabolite). Values of release of lactate were adjusted to cell density upon background subtraction.

\section{Nanopore sequencing}

crRNA guides specific to the regions of interest (ROI) were designed as per recommended guidelines described in the Nanopore infosheet on Targeted, amplification-free DNA sequencing using CRISPR/Cas (Version: ECI_S1014_v1_revA_11Dec2018). The crRNA sequences are provided in Supplementary Table 2. Guides were reconstituted to $100 \mu \mathrm{M}$ using TE ( $\mathrm{pH} 7.5$ ) and pooled into an equimolar mix. For each distinct sample, four identical reactions were prepared parallelly using $5 \mu \mathrm{g}$ gDNA each. Ribonucleoprotein complex (RNPs) assembly, genomic DNA dephosphorylation, and Cas9 cleavage were performed as described in Gilpatrick et al. 2019 (82). Affinity-based Cas9Mediated Enrichment (ACME) using Invitrogen ${ }^{\mathrm{TM}}$ His-Tag Dynabeads ${ }^{\mathrm{TM}}$ was performed to pulldown Cas9 bound non-target DNA, increasing the proportion of on-target reads in the sample (83). The resultant product was cleaned up using 1X Ampure XP beads (Beckman Coulter, Cat \#A63881), eluted in nuclease-free water, and pooled together. The ACME enriched sample was quantified using Qubit fluorometer (ThermoFisher Scientific) and carried forward to the adapter ligation step as described by Iyer et al (83). Sequencing adaptors from the Oxford Nanopore Ligation Sequencing Kit (ONT, SQKLSK109) were ligated to the target fragments using T4 DNA ligase (NEBNext Quick Ligation Module E6056). The sample was cleaned up using 0.3X Ampure XP beads (Beckman Coulter, Cat \#A63881), washed with long-fragment buffer (LFB; ONT, SQK-LSK109), and eluted in $15 \mu$ l of elution buffer (EB; ONT, LSK109) for $30 \mathrm{~min}$ at room temperature. Resultant library was prepared for loading as described in the Cas9-mediated PCR-free enrichment protocol from ONT (Version: ENR_9084_v109_revH_04Dec2018) by adding $25 \mu \mathrm{L}$ sequencing buffer (SQB; ONT, LSK109) and

$13 \mu$ l loading beads (LB; ONT, LSK109) to $12 \mu \mathrm{l}$ of the eluate. Each sample was run on a FLOMIN106 R9.4.1 flow cell using the GridION sequencer. 
Real time basecalling was performed with Guppy v3.2, and files were synced to our Isilon 400NL storage server for further processing on the shared CSHL HPCC. Nanopolish v0.13.2 (84) was used to call methylation per the recommended workflow. Briefly, indexing was performed to match the ONT fastq read IDs with the raw signal level fast5 data. The ONT reads were then aligned to the human reference genome (UCSC hg38) using minimap2 v2.17 (85) and the resulting alignments were then sorted with samtools v0.1.19 (86). Nanopolish call-methylation was then used to detect methylated bases within the targeted regions - specifically 5-methylcytosine in a $\mathrm{CpG}$ context. The initial output file contained the position of the $\mathrm{CpG}$ dinucleotide in the reference genome and the methylation call in each read. A positive value for log_lik_ratio was used to indicate support for methylation, using a cutoff value of 2.0. The helper script calculate_methylation.py was then used to calculate the frequency of methylation calls by genomic position.

\section{qPCR analysis of indel mutations}

To analyze sgRNA editing of genomic DNA as shown in Figure S5A, cells were infected with LRG2.1-sgROSA-GFP-BlastR，LRG2.1-sgSLC5A3-1-GFP-BlastR， or LRG2.1-sgSLC5A3-2-GFPBlastR. At day 2 post infection, blasticidin was added to the cell culture to select for infected cells. After 3 days selection, the cells were collected, and genomic DNA was extracted using QIAamp DNA Mini kit (QIAGEN). For qPCR, 20 25 ng of genomic DNA was used in each reaction with indicated primer set. The $\mathrm{Ct}$ value of non-sgRNA-target primer was used to normalize the data. To analyze SLC5A3 (r-1 and r-2) expression as shown in Figure S6E, MOLM-13 cells were infected with the indicated cDNA constructs, and were then selected with G418 for two weeks. Total RNA was extracted using TRIzol (ThermoFisher Scientific), and cDNA was synthesized using qScript cDNA SuperMix (Quanta). All qPCR experiments were performed using Power SYBR Green PCR Master 
Mix (ThermoFisher Scientific) on a QuantStudio Flex Real-time PCR machine (ThermoFisher Scientific).

\section{AML patient data}

TCGA PanCancer AML patient data, including gene expression and mutation, were downloaded via cBioPortal (cbioportal.org) $(87,88,89)$. The same patients' DNA methylation data was downloaded via NCI Genomic Data Commons (gdc.cancer.gov) (55). The Glass et al data from AML ( $\mathrm{n}=119)$ and normal ( $\mathrm{n}=22$ ) individuals were downloaded from GEO (accession number GSE98350) (56). After filtering and normalizing by coverage, a methylBase object containing the methylation information and locations of cytosines that were present in at least 5 samples per condition (meth.min=5) was generated using MethylKit (version 1.9.3) and R statistical software (version 3.5.1). Percent methylation for each CG for each donor, was calculated using the MethylKit 'percMethylation' function. Bedtools intersect function was used to determine overlap with CpGi from hg19.

\section{ChIP-seq data analysis}

For ChIP-seq analysis shown in Figure S15-A, raw reads were obtained from public GEO datasets, MOLM13 (GSE63782/_GSM1652920), K562 (GSE63782/GSM1652918) (90), MV411 (GSE79899/ GSM2108039), THP-1(GSE79899/ GSM2108046) (91), HEL (GSE109492/ GSM2944364) (42), and mapped to the human genome (hg19) using Bowtie2 software with sensitive settings (92). Duplicate reads were removed prior to peak calling using MACS2 software using 5\% FDR cut off and broad peak option (93). Sequencing depth normalized ChIP-seq pileup tracks were visualization using the IGV genome browser (94).

\section{Statistics}

Error bars represent the mean plus or minus standard error of the mean, and $\mathrm{n}$ refers to the number of 
biological repeats. Statistical significance was evaluated by p value using GraphPad Prism software or Scipy package from Python 3.8.5 as indicated in the figure legends. For Kaplan-Meier survival curves, the log rank (Mantel-Cox) test was used to estimate median overall survival and statistical significance. 


\section{ACKNOWLEDGMENTS}

We thank James C. Mulloy for sharing genetically engineered human AML cell lines. The results analyzing patient data published here are in part based upon data generated by the TCGA Research Network: https://www.cancer.gov/tcga.

\section{AUTHORSHIP CONTRIBUTIONS}

Y.W., Y.H., D.S., S.V.I., A.S.H.C., Z.Y., M.K., E.R.A., O.K., O.E.D., S.P., K.C., S.G., performed experiments and/or analyzed the data; M.E.F., W.R.M, E.H., and C.R.V. supervised the experiments and analysis; Y.W. and C.R.V. wrote the manuscript. 


\section{References}

1. DeBerardinis RJ, Chandel NS. Fundamentals of cancer metabolism. Sci Adv. 2016;2:e1600200.

2. Garcia-Bermudez J, Williams RT, Guarecuco R, Birsoy K. Targeting extracellular nutrient dependencies of cancer cells. Mol Metab. 2020;33:67-82.

3. Possemato R, Marks KM, Shaul YD, Pacold ME, Kim D, Birsoy K, et al. Functional genomics reveal that the serine synthesis pathway is essential in breast cancer. Nature. 2011;476:346-50.

4. Wise DR, DeBerardinis RJ, Mancuso A, Sayed N, Zhang X-Y, Pfeiffer HK, et al. Myc regulates a transcriptional program that stimulates mitochondrial glutaminolysis and leads to glutamine addiction. Proc Natl Acad Sci USA. 2008;105:18782-7.

5. Cramer SL, Saha A, Liu J, Tadi S, Tiziani S, Yan W, et al. Systemic depletion of L-cyst(e)ine with cyst(e)inase increases reactive oxygen species and suppresses tumor growth. Nat Med. 2017;23:120-7.

6. Peng H, Shen N, Qian L, Sun XL, Koduru P, Goodwin LO, et al. Hypermethylation of CpG islands in the mouse asparagine synthetase gene: relationship to asparaginase sensitivity in lymphoma cells. Partial methylation in normal cells. Br J Cancer. 2001;85:930-5.

7. Jaffe N, Traggis D, Das L, Moloney WC, Hann HW, Kim BS, et al. L-asparaginase in the treatment of neoplastic diseases in children. Cancer Res. 1971;31:942-9.

8. Nicholson LJ, Smith PR, Hiller L, Szlosarek PW, Kimberley C, Sehouli J, et al. Epigenetic silencing of argininosuccinate synthetase confers resistance to platinum-induced cell death but collateral sensitivity to arginine auxotrophy in ovarian cancer. Int J Cancer. 2009;125:1454-63

9. Garcia-Bermudez J, Baudrier L, Bayraktar EC, Shen Y, La K, Guarecuco R, et al. Squalene accumulation in cholesterol auxotrophic lymphomas prevents oxidative cell death. Nature. 2019;567:118-22.

10. Nakanishi T, Tamai I. Solute carrier transporters as targets for drug delivery and pharmacological intervention for chemotherapy. J Pharm Sci. 2011;100:3731-50.

11. Bai X, Moraes TF, Reithmeier RAF. Structural biology of solute carrier (SLC) membrane transport proteins. Mol Membr Biol. 2017;34:1-32.

12. Ganapathy V, Thangaraju M, Prasad PD. Nutrient transporters in cancer: relevance to Warburg hypothesis and beyond. Pharmacol Ther. 2009;121:29-40.

13. Bacci M, Lorito N, Ippolito L, Ramazzotti M, Luti S, Romagnoli S, et al. Reprogramming of amino acid transporters to support aspartate and glutamate dependency sustains endocrine resistance in breast cancer. Cell Rep. 2019;28:104-118.e8.

14. El-Gebali S, Bentz S, Hediger MA, Anderle P. Solute carriers (SLCs) in cancer. Mol Aspects Med. 2013;34:719-34.

15. Lin L, Yee SW, Kim RB, Giacomini KM. SLC transporters as therapeutic targets: emerging opportunities. Nat Rev Drug Discov. 2015;14:543-60.

16. Zhang Y, Zhang Y, Sun K, Meng Z, Chen L. The SLC transporter in nutrient and metabolic sensing, regulation, and drug development. J Mol Cell Biol. 2019;11:1-13.

17. Nyquist MD, Prasad B, Mostaghel EA. Harnessing solute carrier transporters for precision oncology. Molecules. 2017;22 (4): 539

18. Hu K, Li K, Lv J, Feng J, Chen J, Wu H, et al. Suppression of the SLC7A11/glutathione axis causes synthetic lethality in KRAS-mutant lung adenocarcinoma. J Clin Invest. 2020;130:175266.

19. Holub BJ. Metabolism and function of myo-inositol and inositol phospholipids. Annu Rev Nutr. 1986;6:563-97.

20. Croze ML, Soulage CO. Potential role and therapeutic interests of myo-inositol in metabolic diseases. Biochimie. 2013;95:1811-27. 
21. Martelli AM, Evangelisti C, Chiarini F, McCubrey JA. The phosphatidylinositol 3kinase/Akt/mTOR signaling network as a therapeutic target in acute myelogenous leukemia patients. Oncotarget. 2010;1:89-103.

22. Berridge MJ, Irvine RF. Inositol phosphates and cell signalling. Nature. 1989;341:197-205.

23. Bansal VS, Majerus PW. Phosphatidylinositol-derived precursors and signals. Annu Rev Cell Biol. 1990;6:41-67.

24. Nakanishi T, Balaban RS, Burg MB. Survey of osmolytes in renal cell lines. Am J Physiol. 1988;255:C181-91.

25. Kitamura H, Yamauchi A, Nakanishi T, Takamitsu Y, Sugiura T, Akagi A, et al. Effects of inhibition of myo-inositol transport on MDCK cells under hypertonic environment. Am J Physiol. 1997;272:F267-72.

26. Eisenberg F, Bolden AH. Biosynthesis of inositol in rat testis homogenate. Biochemical and Biophysical Research Communications. 1963;12:72-7.

27. Hauser G, Finelli VN. The biosynthesis of free and phosphatide myo-inositol from glucose by mammalian tissue slices. J Biol Chem. 1963;238:3224-8.

28. Irvine RF, Schell MJ. Back in the water: the return of the inositol phosphates. Nat Rev Mol Cell Biol. 2001;2:327-38.

29. Schneider S. Inositol transport proteins. FEBS Lett. 2015;589:1049-58.

30. Stein AJ, Geiger JH. The crystal structure and mechanism of 1-L-myo-inositol- 1-phosphate synthase. J Biol Chem. 2002;277:9484-91.

31. Kwon HM, Yamauchi A, Uchida S, Preston AS, Garcia-Perez A, Burg MB, et al. Cloning of the cDNa for a Na+/myo-inositol cotransporter, a hypertonicity stress protein. J Biol Chem. 1992;267:6297-301.

32. Hitomi K, Tsukagoshi N. cDNA sequence for rkST1, a novel member of the sodium iondependent glucose cotransporter family. Biochimica et Biophysica Acta (BBA) - Biomembranes. 1994;1190:469-72.

33. Uldry M, Ibberson M, Horisberger JD, Chatton JY, Riederer BM, Thorens B. Identification of a mammalian $\mathrm{H}(+)$-myo-inositol symporter expressed predominantly in the brain. EMBO J. 2001;20:4467-77.

34. Berry GT, Mallee JJ, Kwon HM, Rim JS, Mulla WR, Muenke M, et al. The human osmoregulatory Na+/myo-inositol cotransporter gene (SLC5A3): molecular cloning and localization to chromosome 21. Genomics. 1995;25:507-13.

35. Hager K, Hazama A, Kwon HM, Loo DD, Handler JS, Wright EM. Kinetics and specificity of the renal Nat/myo-inositol cotransporter expressed in Xenopus oocytes. J Membr Biol. 1995;143:103-13.

36. Roll P, Massacrier A, Pereira S, Robaglia-Schlupp A, Cau P, Szepetowski P. New human sodium/glucose cotransporter gene (KST1): identification, characterization, and mutation analysis in ICCA (infantile convulsions and choreoathetosis) and BFIC (benign familial infantile convulsions) families. Gene. 2002;285:141-8.

37. Coady MJ, Wallendorff B, Gagnon DG, Lapointe J-Y. Identification of a novel Na+/myo-inositol cotransporter. J Biol Chem. 2002;277:35219-24.

38. Rossiter NJ, Huggler KS, Adelmann CH, Keys HR, Soens RW, Sabatini DM, et al. CRISPR screens in physiologic medium reveal conditionally essential genes in human cells. Cell Metab. 2021;33:1248-1263.e9.

39. Lu B, Klingbeil O, Tarumoto Y, Somerville TDD, Huang Y-H, Wei Y, et al. A transcription factor addiction in leukemia imposed by the MLL promoter sequence. Cancer Cell. 2018;34:970981.e8.

40. Brien GL, Remillard D, Shi J, Hemming ML, Chabon J, Wynne K, et al. Targeted degradation of BRD9 reverses oncogenic gene expression in synovial sarcoma. elife. 2018;7. 
Author Manuscript Published OnlineFirst on September 16, 2021; DOI: 10.1158/2159-8290.CD-20-1849

Author manuscripts have been peer reviewed and accepted for publication but have not yet been edited.

41. Lan X, Khandros E, Huang P, Peslak SA, Bhardwaj SK, Grevet JD, et al. The E3 ligase adaptor molecule SPOP regulates fetal hemoglobin levels in adult erythroid cells. Blood Adv. 2019;3:1586-97.

42. Tarumoto Y, Lu B, Somerville TDD, Huang Y-H, Milazzo JP, Wu XS, et al. LKB1, SaltInducible Kinases, and MEF2C Are Linked Dependencies in Acute Myeloid Leukemia. Mol Cell. 2018;69:1017-1027.e6.

43. Grevet JD, Lan X, Hamagami N, Edwards CR, Sankaranarayanan L, Ji X, et al. Domain-focused CRISPR screen identifies HRI as a fetal hemoglobin regulator in human erythroid cells. Science. 2018;361:285-90.

44. Oldenborg PA, Zheleznyak A, Fang YF, Lagenaur CF, Gresham HD, Lindberg FP. Role of CD47 as a marker of self on red blood cells. Science. 2000;288:2051-4.

45. Blazar BR, Lindberg FP, Ingulli E, Panoskaltsis-Mortari A, Oldenborg PA, Iizuka K, et al. CD47 (integrin-associated protein) engagement of dendritic cell and macrophage counterreceptors is required to prevent the clearance of donor lymphohematopoietic cells. J Exp Med. 2001;194:541-9.

46. Sick E, Jeanne A, Schneider C, Dedieu S, Takeda K, Martiny L. CD47 update: a multifaceted actor in the tumour microenvironment of potential therapeutic interest. Br J Pharmacol. 2012;167:1415-30.

47. Burger JA, Kipps TJ. CXCR4: a key receptor in the crosstalk between tumor cells and their microenvironment. Blood. 2006;107:1761-7.

48. Meacham CE, Lawton LN, Soto-Feliciano YM, Pritchard JR, Joughin BA, Ehrenberger T, et al. A genome-scale in vivo loss-of-function screen identifies Phf6 as a lineage-specific regulator of leukemia cell growth. Genes Dev. 2015;29:483-8.

49. Meyers RM, Bryan JG, McFarland JM, Weir BA, Sizemore AE, Xu H, et al. Computational correction of copy number effect improves specificity of CRISPR-Cas9 essentiality screens in cancer cells. Nat Genet. 2017;49:1779-84.

50. Dempster JM, Rossen J, Kazachkova M, Pan J, Kugener G, Root DE, et al. Extracting Biological Insights from the Project Achilles Genome-Scale CRISPR Screens in Cancer Cell Lines. bioRxiv. 2019;720243; doi: https://doi.org/10.1101/720243

51. Berry GT, Wu S, Buccafusca R, Ren J, Gonzales LW, Ballard PL, et al. Loss of murine $\mathrm{Na}+$ /myo-inositol cotransporter leads to brain myo-inositol depletion and central apnea. J Biol Chem. 2003;278:18297-302.

52. Chau JFL, Lee MK, Law JWS, Chung SK, Chung SSM. Sodium/myo-inositol cotransporter-1 is essential for the development and function of the peripheral nerves. FASEB J. 2005;19:1887-9.

53. Moyer JD, Malinowski N, Napier EA, Strong J. Uptake and metabolism of myo-inositol by L1210 leukaemia cells. Biochem J. 1988;254:95-100.

54. Hodges E, Molaro A, Dos Santos CO, Thekkat P, Song Q, Uren PJ, et al. Directional DNA methylation changes and complex intermediate states accompany lineage specificity in the adult hematopoietic compartment. Mol Cell. 2011;44:17-28.

55. Cancer Genome Atlas Research Network, Ley TJ, Miller C, Ding L, Raphael BJ, Mungall AJ, et al. Genomic and epigenomic landscapes of adult de novo acute myeloid leukemia. N Engl J Med. 2013;368:2059-74.

56. Glass JL, Hassane D, Wouters BJ, Kunimoto H, Avellino R, Garrett-Bakelman FE, et al. Epigenetic identity in AML depends on disruption of nonpromoter regulatory elements and is affected by antagonistic effects of mutations in epigenetic modifiers. Cancer Discov. 2017;7:868-83.

57. Livermore TM, Azevedo C, Kolozsvari B, Wilson MSC, Saiardi A. Phosphate, inositol and polyphosphates. Biochem Soc Trans. 2016;44:253-9. 
58. Steger DJ, Haswell ES, Miller AL, Wente SR, O’Shea EK. Regulation of chromatin remodeling by inositol polyphosphates. Science. 2003;299:114-6.

59. Szlosarek PW, Klabatsa A, Pallaska A, Sheaff M, Smith P, Crook T, et al. In vivo loss of expression of argininosuccinate synthetase in malignant pleural mesothelioma is a biomarker for susceptibility to arginine depletion. Clin Cancer Res. 2006;12:7126-31.

60. Delage B, Luong P, Maharaj L, O'Riain C, Syed N, Crook T, et al. Promoter methylation of argininosuccinate synthetase-1 sensitises lymphomas to arginine deiminase treatment, autophagy and caspase-dependent apoptosis. Cell Death Dis. 2012;3:e342.

61. Ren Y, Roy S, Ding Y, Iqbal J, Broome JD. Methylation of the asparagine synthetase promoter in human leukemic cell lines is associated with a specific methyl binding protein. Oncogene. 2004;23:3953-61.

62. Touzart A, Lengliné E, Latiri M, Belhocine M, Smith C, Thomas X, et al. Epigenetic Silencing Affects 1-Asparaginase Sensitivity and Predicts Outcome in T-ALL. Clin Cancer Res. 2019;25:2483-93.

63. Ward PS, Patel J, Wise DR, Abdel-Wahab O, Bennett BD, Coller HA, et al. The common feature of leukemia-associated IDH1 and IDH2 mutations is a neomorphic enzyme activity converting alpha-ketoglutarate to 2-hydroxyglutarate. Cancer Cell. 2010;17:225-34.

64. Figueroa ME, Abdel-Wahab O, Lu C, Ward PS, Patel J, Shih A, et al. Leukemic IDH1 and IDH2 mutations result in a hypermethylation phenotype, disrupt TET2 function, and impair hematopoietic differentiation. Cancer Cell. 2010;18:553-67.

65. Yu W, Ye C, Greenberg ML. Inositol hexakisphosphate kinase 1 (IP6K1) regulates inositol synthesis in mammalian cells. J Biol Chem. 2016;291:10437-44.

66. Zhou L, Sheng W, Jia C, Shi X, Cao R, Wang G, et al. Musashi2 promotes the progression of pancreatic cancer through a novel ISYNA1-p21/ZEB-1 pathway. J Cell Mol Med. 2020;24:10560-72.

67. Koguchi T, Tanikawa C, Mori J, Kojima Y, Matsuda K. Regulation of myo-inositol biosynthesis by p53-ISYNA1 pathway. Int J Oncol. 2016;48:2415-24.

68. Egler RA, Ahuja SP, Matloub Y. L-asparaginase in the treatment of patients with acute lymphoblastic leukemia. J Pharmacol Pharmacother. 2016;7:62-71.

69. Shiue E, Prather KLJ. Improving D-glucaric acid production from myo-inositol in E. coli by increasing MIOX stability and myo-inositol transport. Metab Eng. 2014;22:22-31.

70. Zheng S, Hou J, Zhou Y, Fang H, Wang T-T, Liu F, et al. One-pot two-strain system based on glucaric acid biosensor for rapid screening of myo-inositol oxygenase mutations and glucaric acid production in recombinant cells. Metab Eng. 2018;49:212-9.

71. Ramaley R, Fujita Y, Freese E. Purification and properties of Bacillus subtilis inositol dehydrogenase. J Biol Chem. 1979;254:7684-90.

72. Kouzuma T, Takahashi M, Endoh T, Kaneko R, Ura N, Shimamoto K, et al. An enzymatic cycling method for the measurement of myo-inositol in biological samples. Clin Chim Acta. 2001;312:143-51.

73. Mulloy JC, Wunderlich M, Zheng Y, Wei J. Transforming human blood stem and progenitor cells: a new way forward in leukemia modeling. Cell Cycle. 2008;7:3314-9.

74. Wunderlich M, Mizukawa B, Chou F-S, Sexton C, Shrestha M, Saunthararajah Y, et al. AML cells are differentially sensitive to chemotherapy treatment in a human xenograft model. Blood. 2013;121:e90-7.

75. Tarumoto Y, Lin S, Wang J, Milazzo JP, Xu Y, Lu B, et al. Salt-inducible kinase inhibition suppresses acute myeloid leukemia progression in vivo. Blood. 2020;135:56-70.

76. Shi J, Wang E, Milazzo JP, Wang Z, Kinney JB, Vakoc CR. Discovery of cancer drug targets by CRISPR-Cas9 screening of protein domains. Nat Biotechnol. 2015;33:661-7. 
77. Adamson B, Norman TM, Jost M, Cho MY, Nuñez JK, Chen Y, et al. A Multiplexed Single-Cell CRISPR Screening Platform Enables Systematic Dissection of the Unfolded Protein Response. Cell. 2016;167:1867-1882.e21.

78. Li W, Xu H, Xiao T, Cong L, Love MI, Zhang F, et al. MAGeCK enables robust identification of essential genes from genome-scale CRISPR/Cas9 knockout screens. Genome Biol. 2014;15:554.[old 72]

79. Li W, Köster J, Xu H, Chen C-H, Xiao T, Liu JS, et al. Quality control, modeling, and visualization of CRISPR screens with MAGeCK-VISPR. Genome Biol. 2015;16:281.[01d 73

80. Mackay GM, Zheng L, van den Broek NJF, Gottlieb E. Analysis of Cell Metabolism Using LCMS and Isotope Tracers. Meth Enzymol. 2015;561:171-96.

81. Su X, Lu W, Rabinowitz JD. Metabolite spectral accuracy on orbitraps. Anal Chem. 2017;89:5940-8.

82. Gilpatrick T, Lee I, Graham JE, Raimondeau E, Bowen R, Heron A, et al. Targeted nanopore sequencing with Cas9-guided adapter ligation. Nat Biotechnol. 2020;38:433-8.

83. Iyer SV, Goodwin S, Kramer M, McCombie WR. Understanding genetic variation in cancer using targeted nanopore long read sequencing [abstract]. In: Proceedings of the Annual Meeting of the American Association for Cancer Research 2020; 2020 Apr 27-28 and Jun 22-24; Philadelphia (PA): AACR; 2020. Abstract nr 1360.

84. Simpson JT, Workman RE, Zuzarte PC, David M, Dursi LJ, Timp W. Detecting DNA cytosine methylation using nanopore sequencing. Nat Methods. 2017;14:407-10.

85. Li H. Minimap2: pairwise alignment for nucleotide sequences. Bioinformatics. 2018;34:3094100.

86. Li H, Handsaker B, Wysoker A, Fennell T, Ruan J, Homer N, et al. The Sequence Alignment/Map format and SAMtools. Bioinformatics. 2009;25:2078-9.

87. Cerami E, Gao J, Dogrusoz U, Gross BE, Sumer SO, Aksoy BA, et al. The cBio cancer genomics portal: an open platform for exploring multidimensional cancer genomics data. Cancer Discov. 2012;2:401-4.

88. Gao J, Aksoy BA, Dogrusoz U, Dresdner G, Gross B, Sumer SO, et al. Integrative analysis of complex cancer genomics and clinical profiles using the cBioPortal. Sci Signal. 2013;6:pl1.

89. Hoadley KA, Yau C, Hinoue T, Wolf DM, Lazar AJ, Drill E, et al. Cell-of-Origin Patterns Dominate the Molecular Classification of 10,000 Tumors from 33 Types of Cancer. Cell. 2018;173:291-304.e6.

90. Rathert P, Roth M, Neumann T, Muerdter F, Roe J-S, Muhar M, et al. Transcriptional plasticity promotes primary and acquired resistance to BET inhibition. Nature. 2015;525:543-7.

91. Prange KHM, Mandoli A, Kuznetsova T, Wang SY, Sotoca AM, Marneth AE, et al. MLL-AF9 and MLL-AF4 oncofusion proteins bind a distinct enhancer repertoire and target the RUNX1 program in 11q23 acute myeloid leukemia. Oncogene. 2017;36:3346-56.

92. Langmead B, Salzberg SL. Fast gapped-read alignment with Bowtie 2. Nat Methods. 2012;9:357-9.

93. Feng J, Liu T, Qin B, Zhang Y, Liu XS. Identifying ChIP-seq enrichment using MACS. Nat Protoc. 2012;7:1728-40.

94. Robinson JT, Thorvaldsdóttir H, Winckler W, Guttman M, Lander ES, Getz G, et al. Integrative genomics viewer. Nat Biotechnol. 2011;29:24-6.

95. Hart T, Chandrashekhar M, Aregger M, Steinhart Z, Brown KR, MacLeod G, et al. HighResolution CRISPR Screens Reveal Fitness Genes and Genotype-Specific Cancer Liabilities. Cell. 2015;163:1515-26.

96. Ghandi M, Huang FW, Jané-Valbuena J, Kryukov GV, Lo CC, McDonald ER, et al. Nextgeneration characterization of the Cancer Cell Line Encyclopedia. Nature. 2019;569:503-8. 
97. Barretina J, Caponigro G, Stransky N, Venkatesan K, Margolin AA, Kim S, et al. The Cancer Cell Line Encyclopedia enables predictive modelling of anticancer drug sensitivity. Nature. 2012;483:603-7. 


\section{FIGURE LEGENDS}

Figure 1. A domain-focused CRISPR screening method for identifying in vivo-relevant AML dependencies. (A) Overview of in vivo genetic screening procedure using domain-focused sgRNA libraries. GPCR, G-protein coupled receptor; NGS, Next Gen Sequencing. The numbers represent the number of genes in each sgRNA library. Figures for mouse bone marrow, spleen and NGS were created with a licensed version of BioRender . (B-E) Examples of in vivo CRISPR screening results with sgRNA libraries targeting kinases (B), transcription factors (C), chromatin regulators (D), and membrane proteins (E). The gene beta score was calculated using MAGeCK $(78,79)$. The beta score describes how the gene knockout alters cell fitness: a positive beta score indicates positive selection, and a negative beta score indicates negative selection. The screening results using pooled spleen samples were compared with in vitro screening results. Core fitness genes (95) are labeled in red, negative control sgRNAs are labeled in blue. (F) Venn diagram comparing genetic dependencies identified by in vivo screening with in vitro screening. The in vivo-relevant targets were determined using spleen data with $p$-value $<0.05$, FDR $<0.25$, beta score $<0$. For in vivo- and in vitro-specific targets, the absolute value of differential beta scores were shown as pie charts. Genes with a differential beta score of 2 or greater are listed.

Figure 2. Dependency on SLC5A3 is in vivo-relevant and AML-specific. (A) Analysis of Project Achilles genetic screening data, evaluating the leukemia-biased essentiality of 239 in vivo-relevant dependencies. The in vivo screening dependencies were determined by $p$-value $<0.05$, FDR $<0.25$, beta score $<0$. The average gene effect scores were calculated using Achilles Gene Effect 20Q3 data. The $p$ values compared gene effect scores in leukemia vs. other cancers using an unpaired Student's t-test. (B) Comparison of dependencies of top SLC candidates between leukemia and other type of cancers. The dependency of SLC5A3 is the most leukemia-biased among the candidates. Data is obtained from DepMap database (20Q3). Plotted as average gene effect score with standard deviation in leukemia 
cell lines vs. non-leukemia cell lines. The $p$ values were calculated using unpaired Student's t-test. (* $p$-value <0.05) (C) Summary of in vivo vs in vitro CRISPR screening results of the SLC sgRNA library.

Figure 3. Validation of SLC5A3 dependency. (A) Summary of in vitro validation experiments of individual sgRNAs (linked to GFP) in competition-based proliferation assays in AML cell lines. Plotted is the $\log 2$ transformed fold change of GFP positive cell percentage at day 18 vs. day 3 post the infection of sgRNA lentivirus. Shown as mean with standard deviation of 2 7 biological replicates. (B) Procedure of in vivo validation of SLC5A3 dependency in human AML. MOLM-13 cells expressing Cas9 and Luciferase were transduced with sgROSA or sgSLC5A3-2 (linked to GFP/bsr), and then selected with Blasticidin. The SLC5A3 knock-out cells (sgSLC5A3) and control cells (sgROSA) were then transplanted into NSG mice via tail vein injection. (C) Bioluminescence imaging of NSG mice transplanted with MOLM-13 cells transduced with the indicated sgRNA. The images were taken on day 13, 16, and 19 post-transplantation. (D) Quantification of bioluminescence from (C). Values represent total flux as photons per second (Mean \pm Standard Deviation). The $p$-value was calculated using unpaired Student's t test $(n=5)$. (E) Survival curves of mice shown in (C). The $p$-value was calculated using Log-rank (Mantel-Cox) test $(\mathrm{n}=5)$.

Figure 4. SLC5A3-dependent AML lines are myo-inositol auxotrophs. (A) Liquid Chromatography Mass Spectrometry (LC-MS) analysis of cellular myo-inositol and glutamine levels in MOLM-13 and MV4-11 cells transduced with indicated sgRNA. The $p$-value was calculated using unpaired Student's t test $(\mathrm{n}=6)$. $(* * * * p<0.0001)$. (B-C) Depletion of myo-inositol in the cell culture medium affects the growth of SLC5A3-dependent lines MOLM-13, MV4-11, and U937 (B), but not SLC5A3-independent lines HEL, K562, and SET-2 (C). A customized RPMI cell culture medium without myo-inositol, supplied with dialyzed FBS, was used (-MI). Additional myo-inositol supplement (1.11 mM/0.2 g/L) 
was added into the medium for rescue (+MI). (Shown as mean with standard deviation of 2-4 biological replicates. The $p$ values were calculated using 2way ANOVA Sidak test to compare each line graph). (D) Competition-based proliferation assays in MOLM13 and MV4-11 cells transduced with indicated sgRNA, and treated with additional myo-inositol supplement. The base level of myoinositol in RPMI medium is $0.035 \mathrm{~g} / \mathrm{L}(0.19 \mathrm{mM})$. To rescue the phenotype, additional myo-inositol was added to the cell culture to make myo-inositol concentration as $0.07 \mathrm{~g} / \mathrm{L}(0.38 \mathrm{mM})$ and $0.175 \mathrm{~g} / \mathrm{L}$ (0.95 mM). Myo-inositol from FBS (100\% FBS contains $\sim 0.9 \mathrm{mM}$, determined by LC-MS, 10\% FBS was added to cell culture medium) was not included. (Shown as mean with standard deviation of 3 replicates with independent infections).

\section{Figure 5. Transcriptional silencing of ISYNA1 leads to SLC5A3 dependency through a synthetic}

lethal genetic interaction. (A) Summary of two major sources of cellular myo-inositol: transported from extracellular environment by SLC5A3; de novo biosynthesis from glucose. The rate limiting denovo myo-inositol biosynthesis enzyme is inositol-3-phosphate synthase 1, encoded by the ISYNAI gene. (B) SLC5A3 dependency in AML (and K562) cell lines correlates with ISYNA1 expression. Data from DepMap database (Achilles 20Q3). (C) Expression of ISYNA1 in AML (and K562) cell lines. The ISYNA1 gene mRNA expression data was obtained from Cancer Cell Line Encyclopedia (CCLE) database $(96,97)$. The ISYNA1 protein expression in AML lines and K562 (CML) was detected by Western blot, shown as one of two replicates. (D) A double knock-out construct that cotargets two genes by two sgRNAs driven by human U6 (hU6) or bovine U6 (bU6) promoter, respectively. The sgRNAs are linked to GFP. (E) Competition-based proliferation assays in Cas9 expressing SLC5A3-independent cell lines, following infection with indicated co-targeting sgRNA constructs. Double knock-out both ISYNA1 and SLC5A3 leads to growth phenotype (red). (Shown as mean with standard deviation of 3 replicates of independent infections.). (F) Western blot shows the depletion of ISYNA1 protein in HEL and K562 cells following infection with indicated sgRNA. (G) 
Competition-based proliferation assays in Cas9 expressing HEL and K562 cells following infection with indicated sgRNA. The experiment was performed in myo-inositol deficient RPMI medium without myo-inositol supplement (-MI, blue), or with $0.05 \mathrm{~g} / \mathrm{L}(0.28 \mathrm{mM})$ myo-inositol supplement (+MI, red). (Shown as mean with standard deviation of 3 replicates with independent infections.)

\section{Figure 6. Re-expression of ISYNA1 eliminates SLC5A3 dependency and the myo-inositol}

auxotrophy. (A) Western blot shows overexpression of flag-tagged ISYNA1 in indicated AML cell lines. (B) Competition-based proliferation assays in SLC5A3-dependent AML lines expressing EV (empty vector, blue) or ISYNA1(red), following infection with indicated sgRNA linked to GFP. (Shown as mean with standard deviation of 3 replicates from independent infections). (C) LC-MS analysis in MOLM-13 cells expressing EV or ISYNA1, following infection with indicated sgRNA. The $p$-value was calculated using 2 way ANOVA $(\mathrm{n}=5) .(* * p=0.0011$, **** $p<0.0001)$. (D) Overexpression of ISYNA1 in MOLM-13 cells rescues the growth defect in myo-inositol deficient medium (-MI), but has no impact on the cell growth in medium with myo-inositol supplement (+MI). (Shown as mean with standard deviation of two biological replicates, each includes 3 replicates with independent infection. The $p$ value was calculated using 2way ANOVA Sidak test.)

Figure 7. The ISYNA1 gene is epigenetically silenced by aberrant DNA methylation in a subset of AML. (A-B) CpG methylation status at the ISYNA1 gene locus in AML cell lines (MOLM-13, MV411, HEL), normal hematopoietic stem and progenitor cell (HSPC) (A), and AML patients (B). The AML cell line methylation was measured by Nanopore sequencing. The HSPC methylation was measured by genome-wide bisulfite sequencing, obtained from a previous study (54). The AML patients' data was obtained from TCGA PanCancer AML database (55). The TCGA AML patients' data used DNA methylation array with reduced $\mathrm{CpG}$ representation, shown as 'TCGA CpG' (blue dots). The CpGs at ISYNA1 promoter (dark blue dots in TCGA CpG) show hypermethylated in 
SLC5A3-dependent/ISYNA1-low AML lines MOLM-13 and MV4-11, but hypomethylated in SLC5A3-independent/ISYNA1-high HEL line as well as normal HSPC cells. These regions were also shown aberrantly methylated in a subset of AML patients (B). (C) ISYNA1 gene expression level is significantly lower in high methylation group, compared to low methylation group $(p=0.02)$. The highand low-groups were determined by the $\mathrm{Z}$ scores of average DNA methylation levels of the three promoter CpGs (TCGA) described above (Figure S15E). (D) CEBPA, IDH1, and IDH2 mutations are enriched in the high ISYNA1 methylation group, while DNMT3A mutation is enriched in low methylation group. Data was obtained from TCGA PanCancer AML database via cBioportal. (E) AML patients with IDH2 mutations show lower ISYNA1 gene expression $(p=0.03)$. Data was obtained from TCGA PanCancer AML database via cBioportal. (F) A model depicting synthetic lethality between SLC5A3 and ISYNA1 in AML. 
A Solute carrierAuthor Wandscript Pubtished

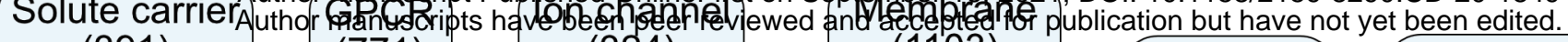

(391)

(771) (324)

(1103)

Chromatin
regulator
$(542)$

Transcription
factor
$(1426)$

(523)

(481)

Glycosyltransferase (345)

MOLM-13-

Cas9-Luc

Domain focused sgRNA

\section{Pooled Genomic DNA}

PCR sgRNA

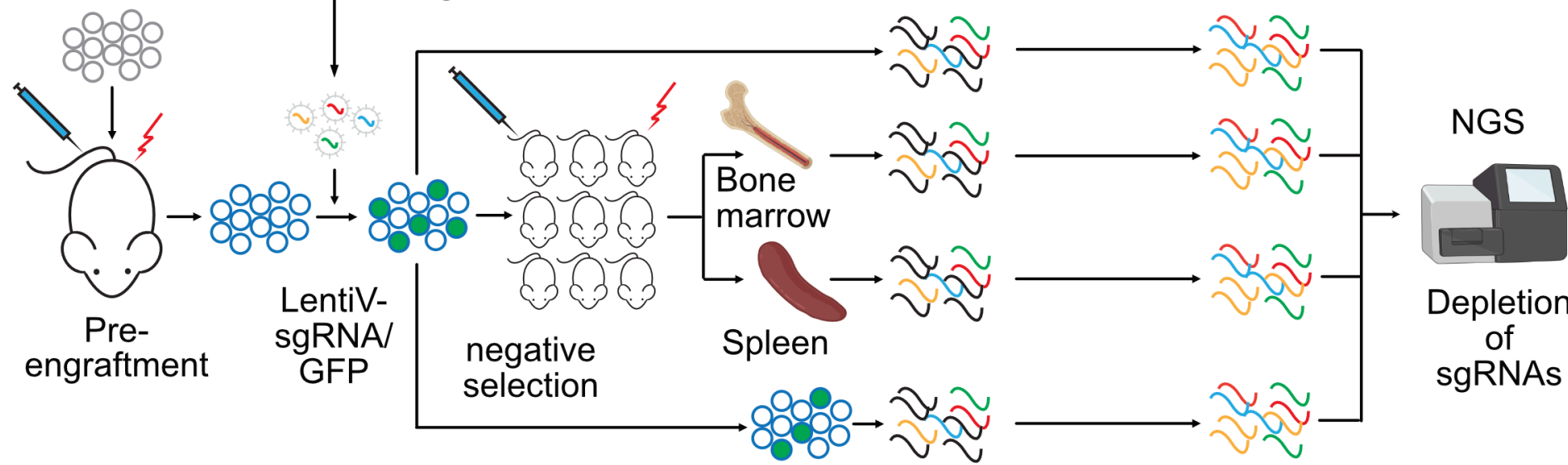

B
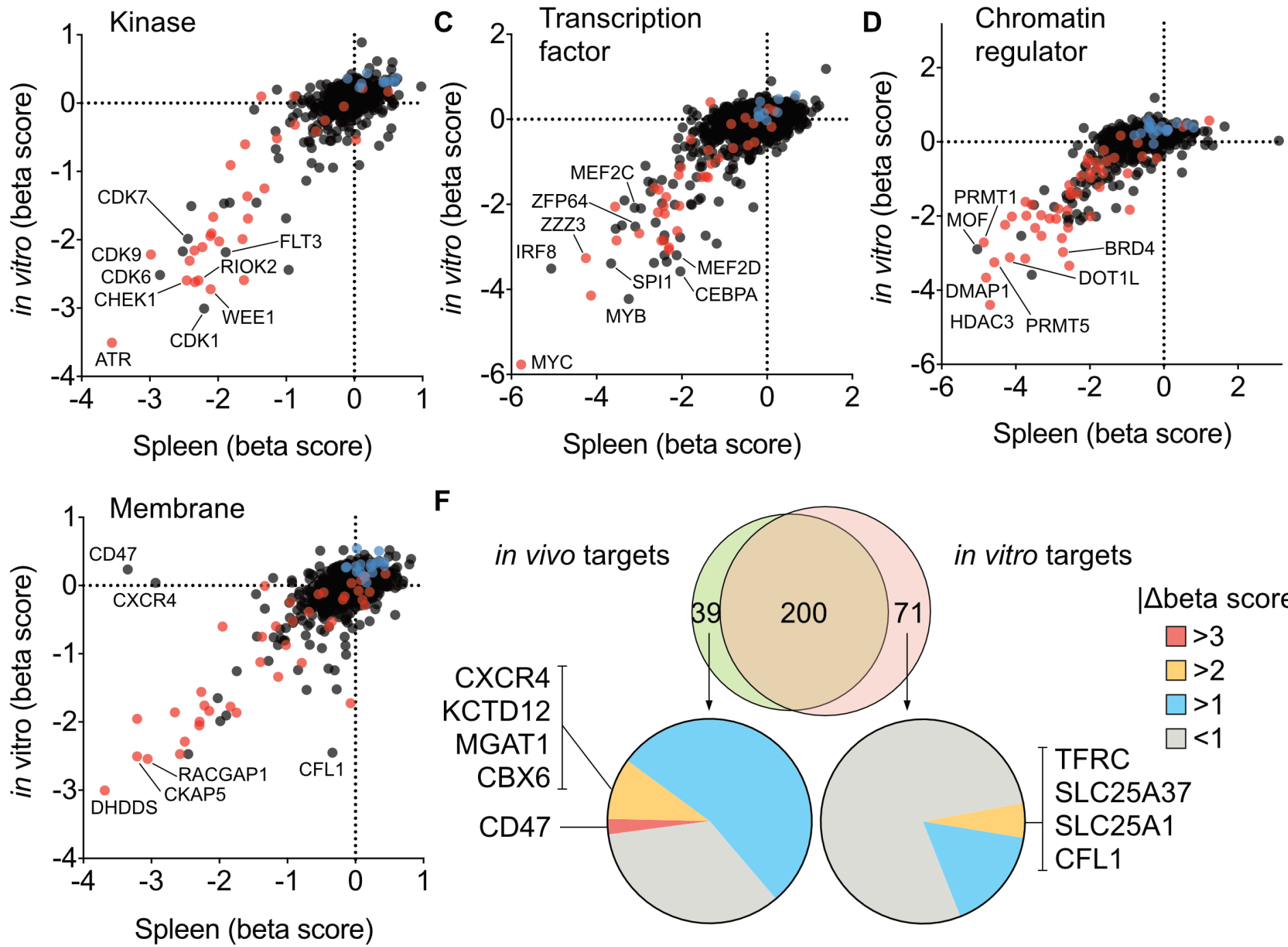

$\mathbf{F}$

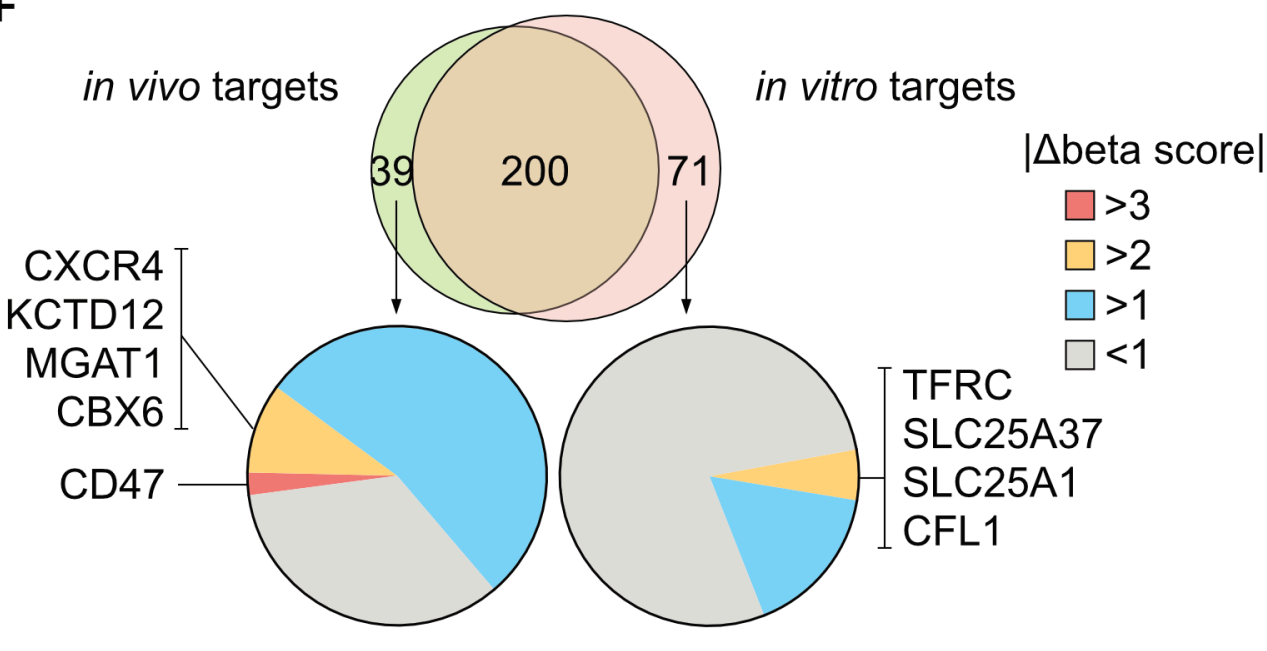

- Others $\bigcirc$ Core fitness $\bigcirc$ Negative control

Targeted genes 
A Author Manuscript Published OnGeFirst on September 16, 2021; DOI: 10.1158/2159-8290.CD-20-1849

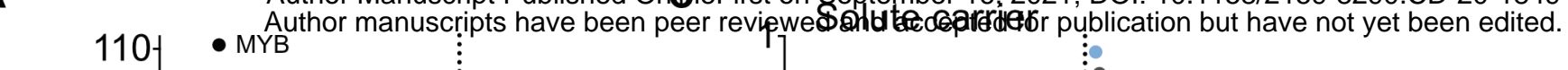
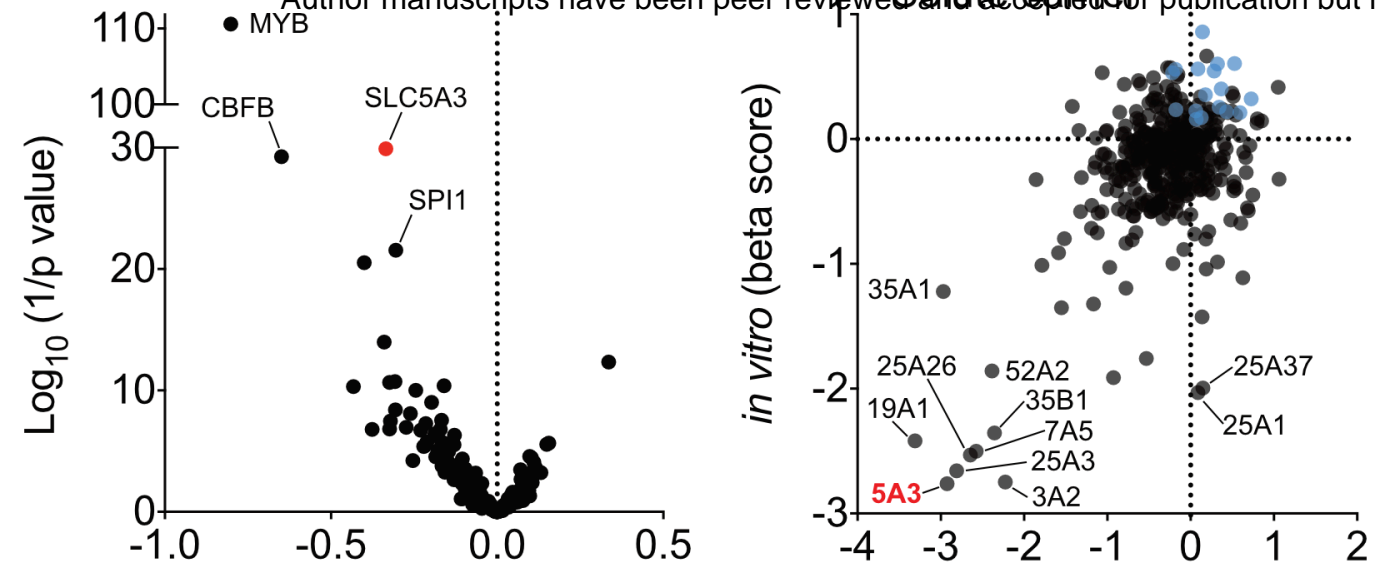

$\triangle$ Avg. Dependency (Leukemia-Other Cancers)

B
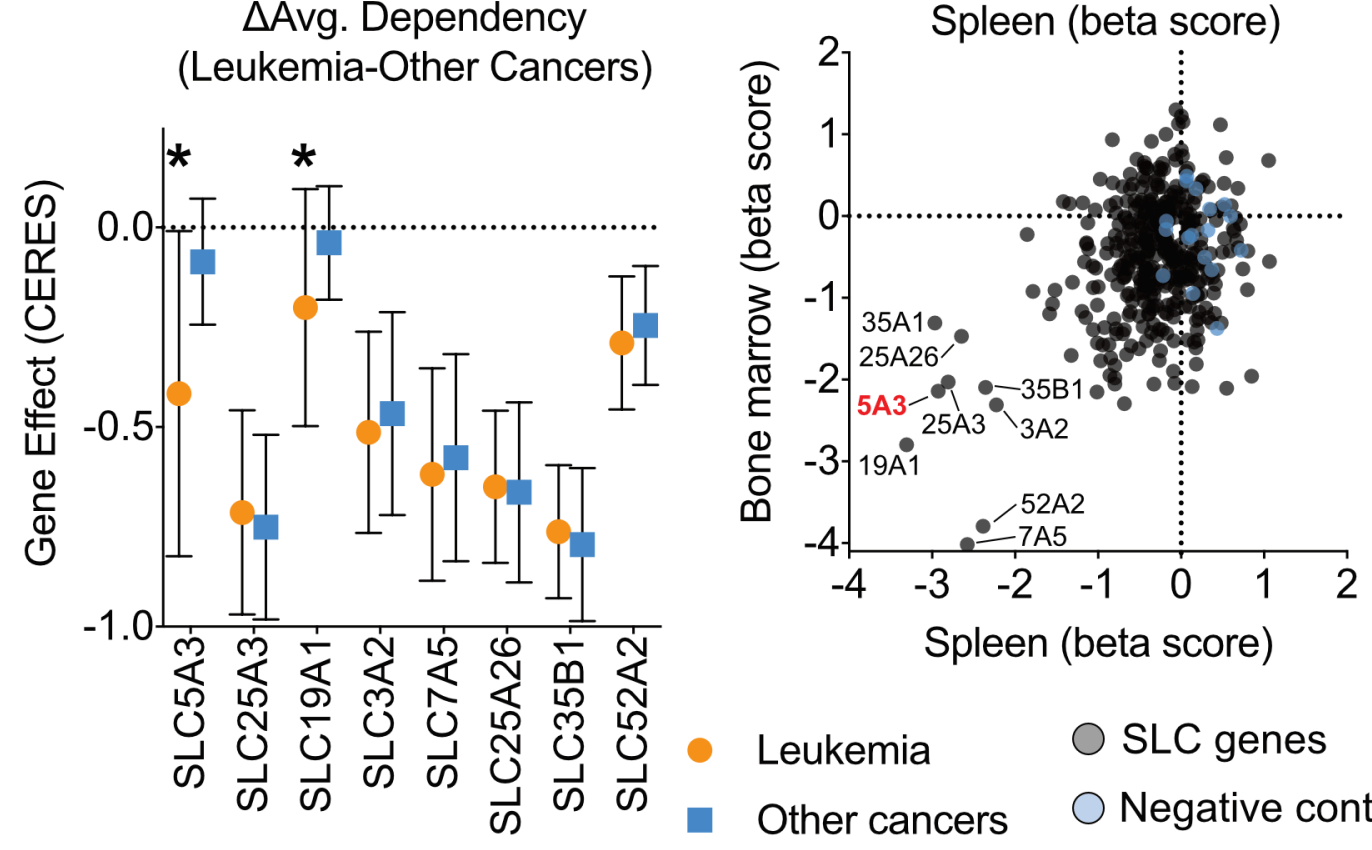

Leukemia

SLC genes

Other cancers

$\bigcirc$ Negative control 
A sgSLC5A A Suthor Manuscript PublisłBd OnlineFirst on September 16, 2021; DOI: 10.1158/21 D9-8290.CD-20-1849

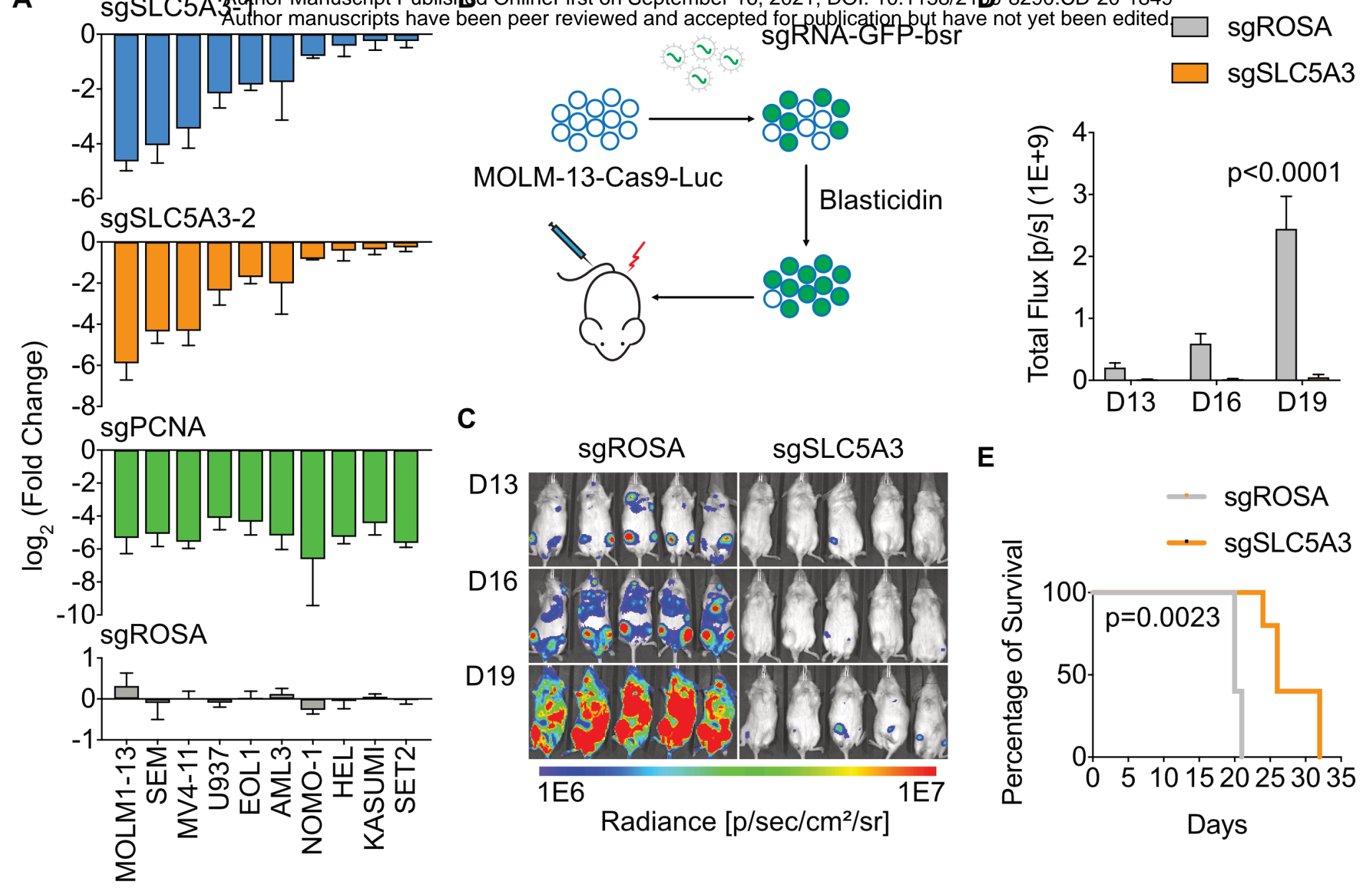




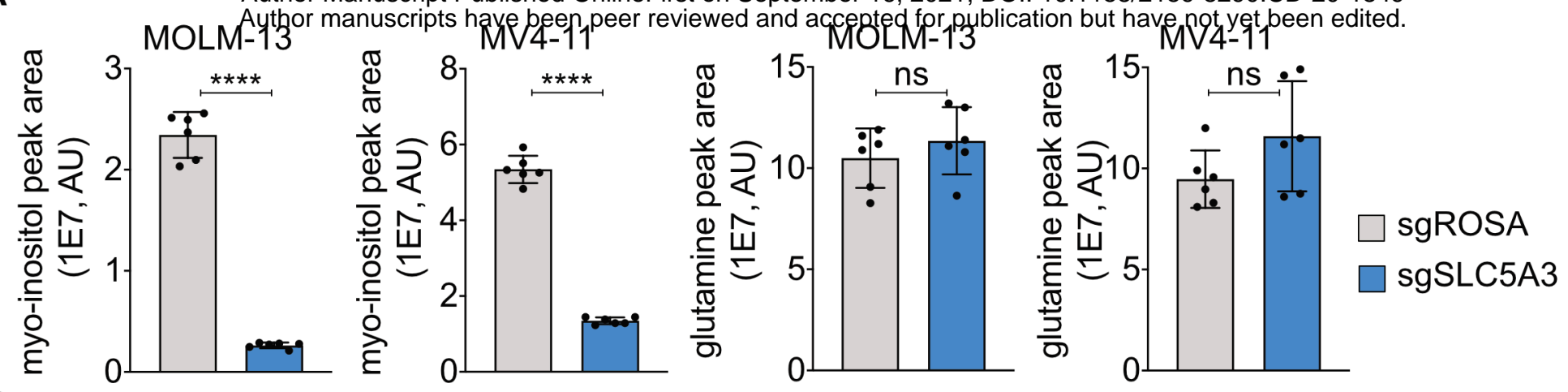

B

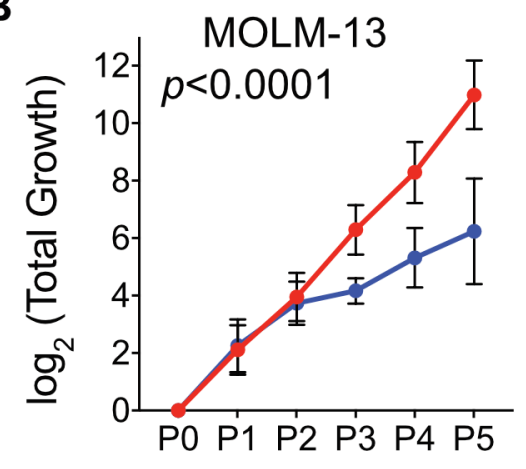

C

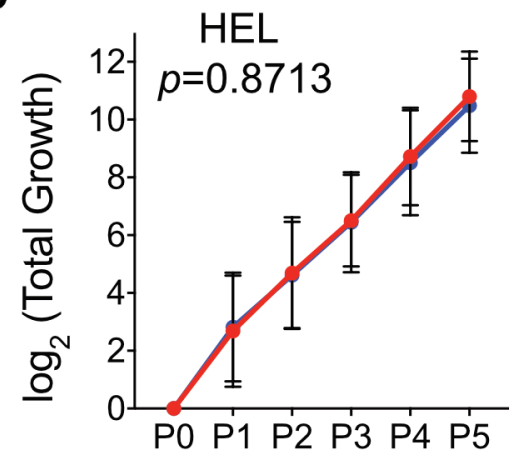

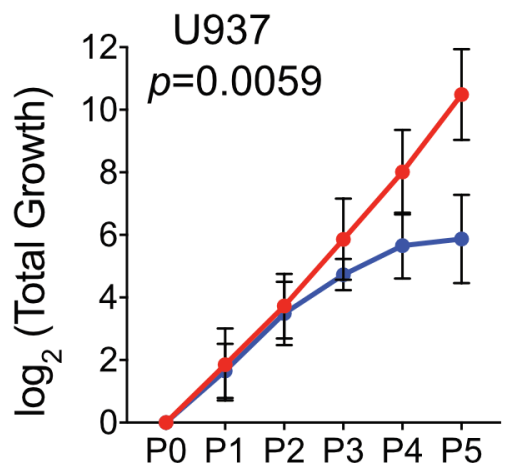

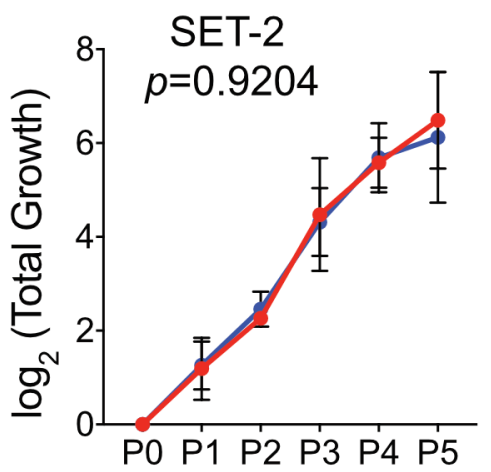

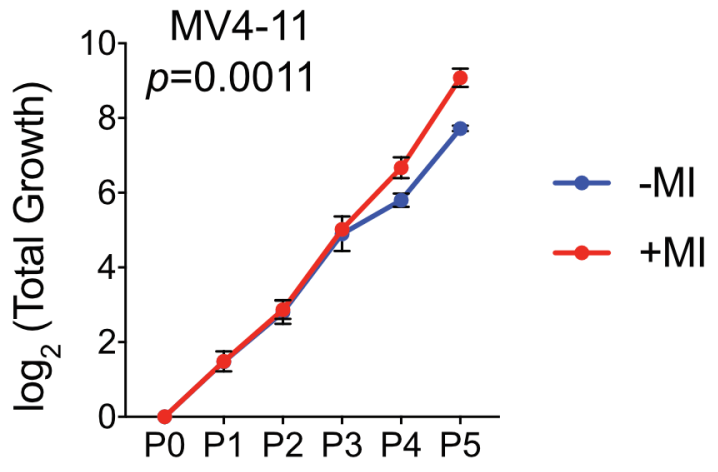

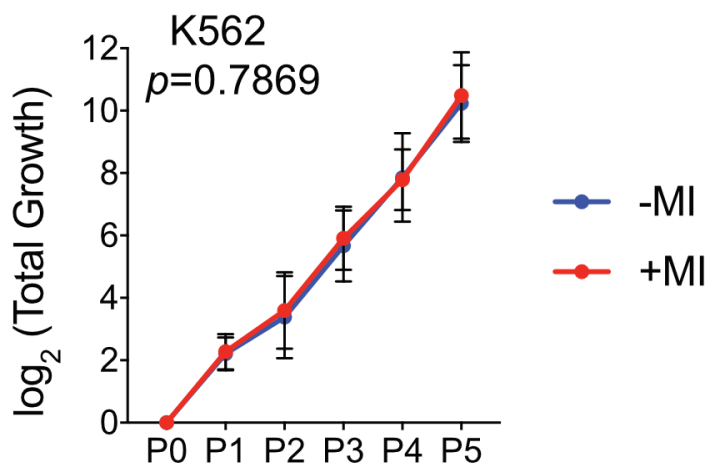

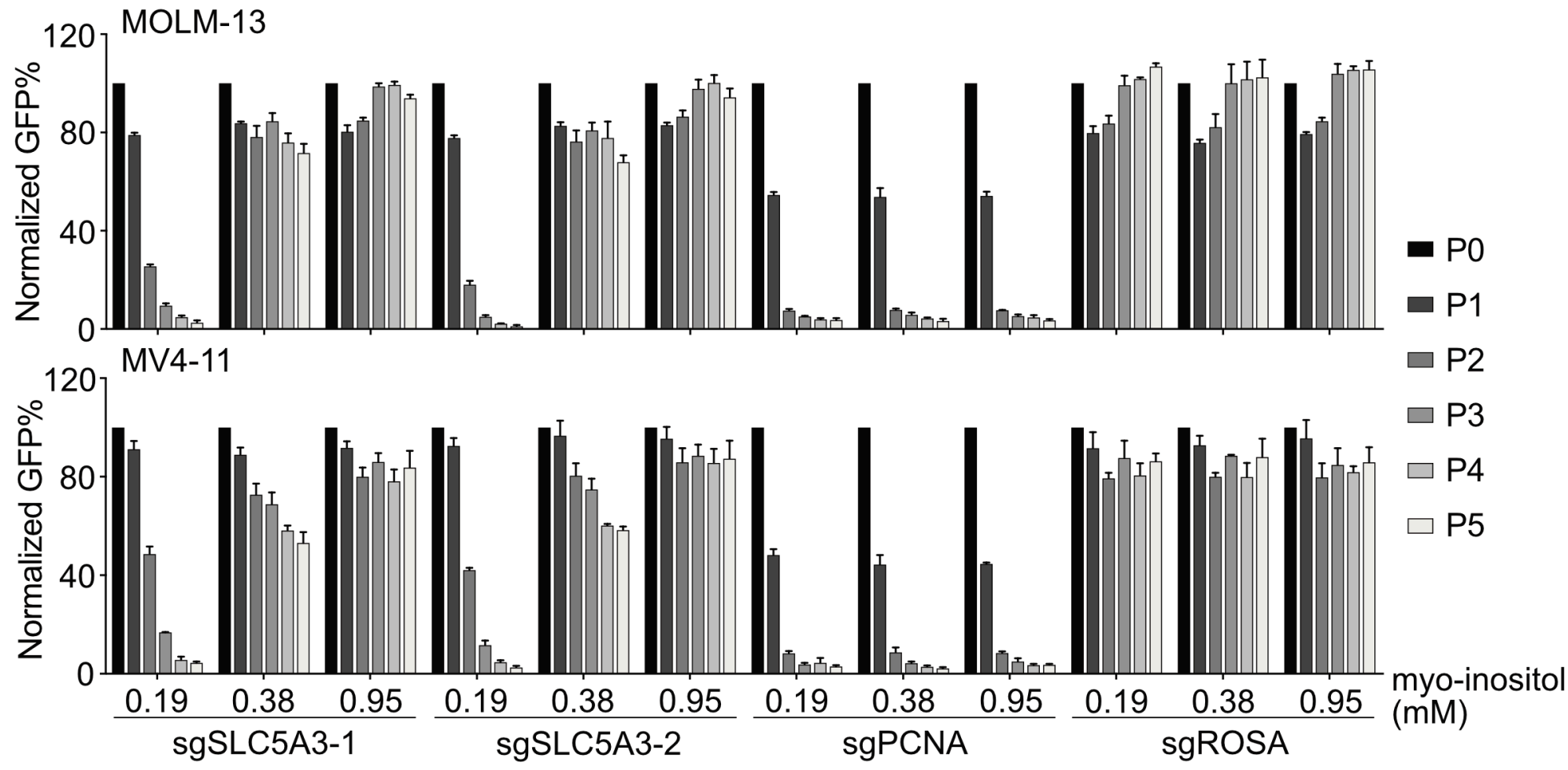


Author Mappuscript Published OnlineFirst on September 16, 2021; DOI: $10.1158 / 2159-8290 . C D-20-1849$
Author manuscripts have been peer reviewed and accepted for publication but have not yet been edited.

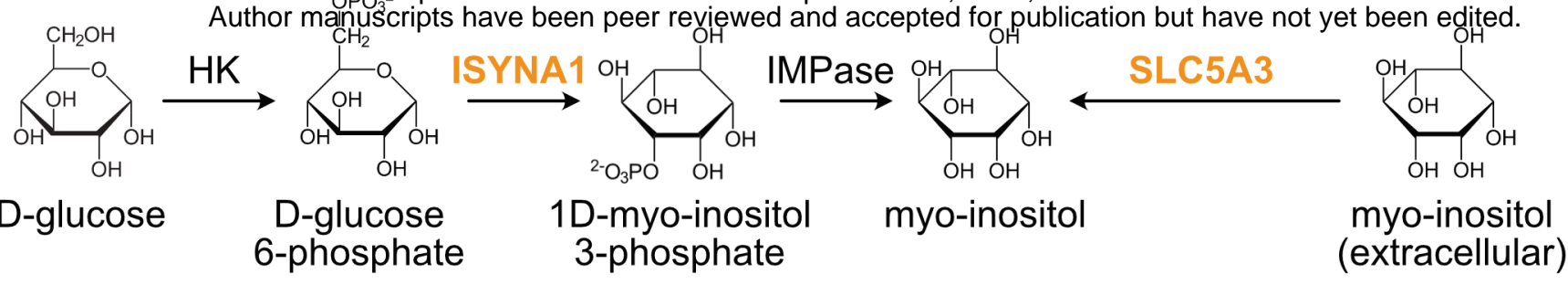

B

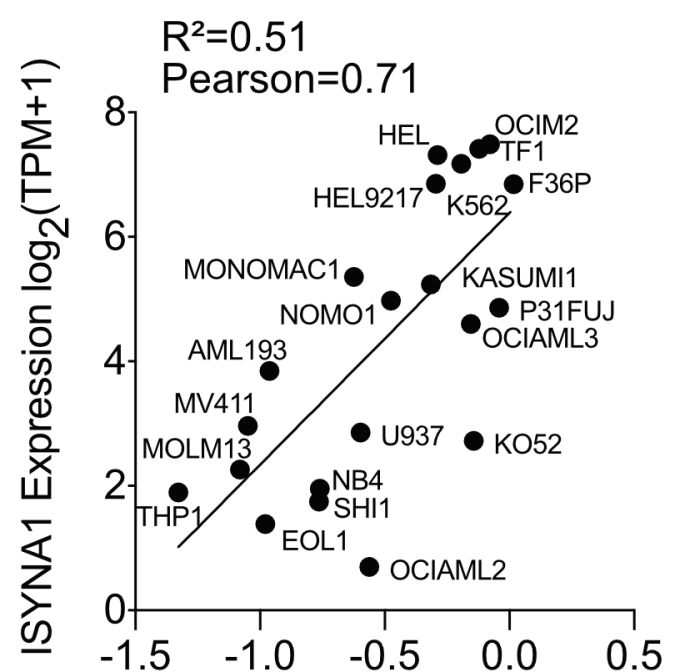

SLC5A3 Dependency Score

C

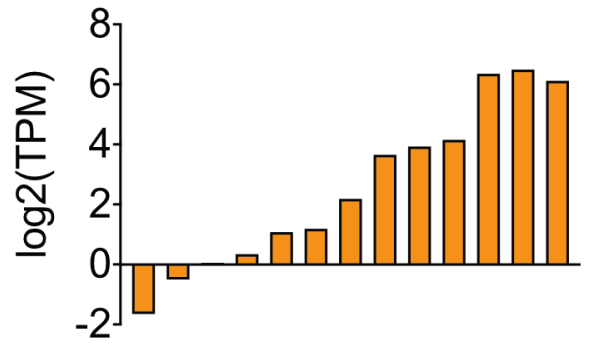

F

$70 \mathrm{kDa}$

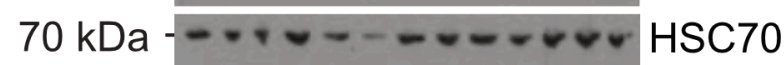

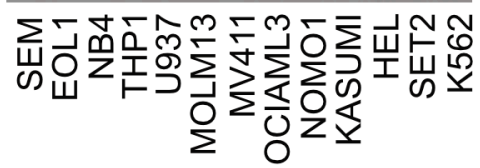
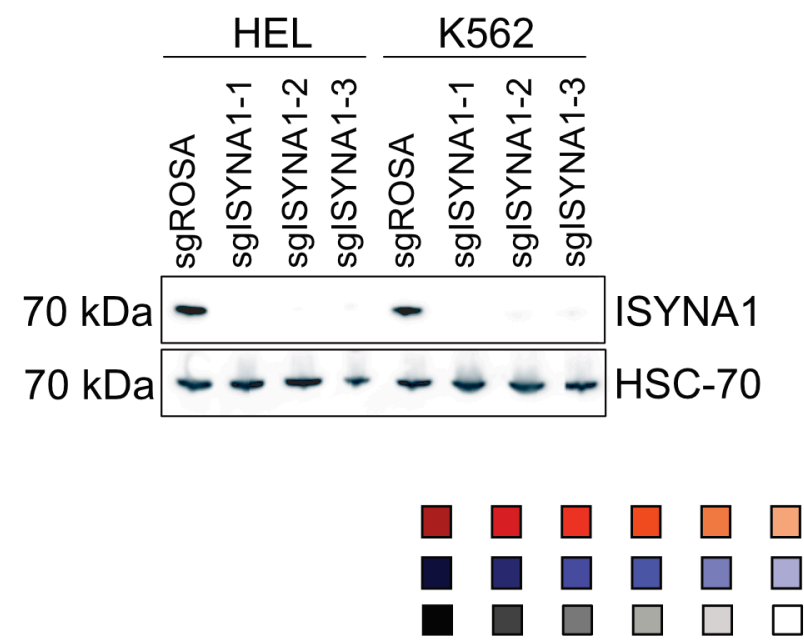

$\begin{array}{lllllll}\text { P0 } & \text { P1 } & \text { P2 } & \text { P3 } & \text { P4 } & \text { P5 }\end{array}$
D $\overrightarrow{\mathrm{hU}} 6$ sgRNA1 $\overrightarrow{\mathrm{bU}} 6$ sgRNA2 $\overrightarrow{\mathrm{EF} S}$ GFP

E

sgRNA1 sgSLC5A3 sgISYNA1 sgROSA sgPCNA sgROSA
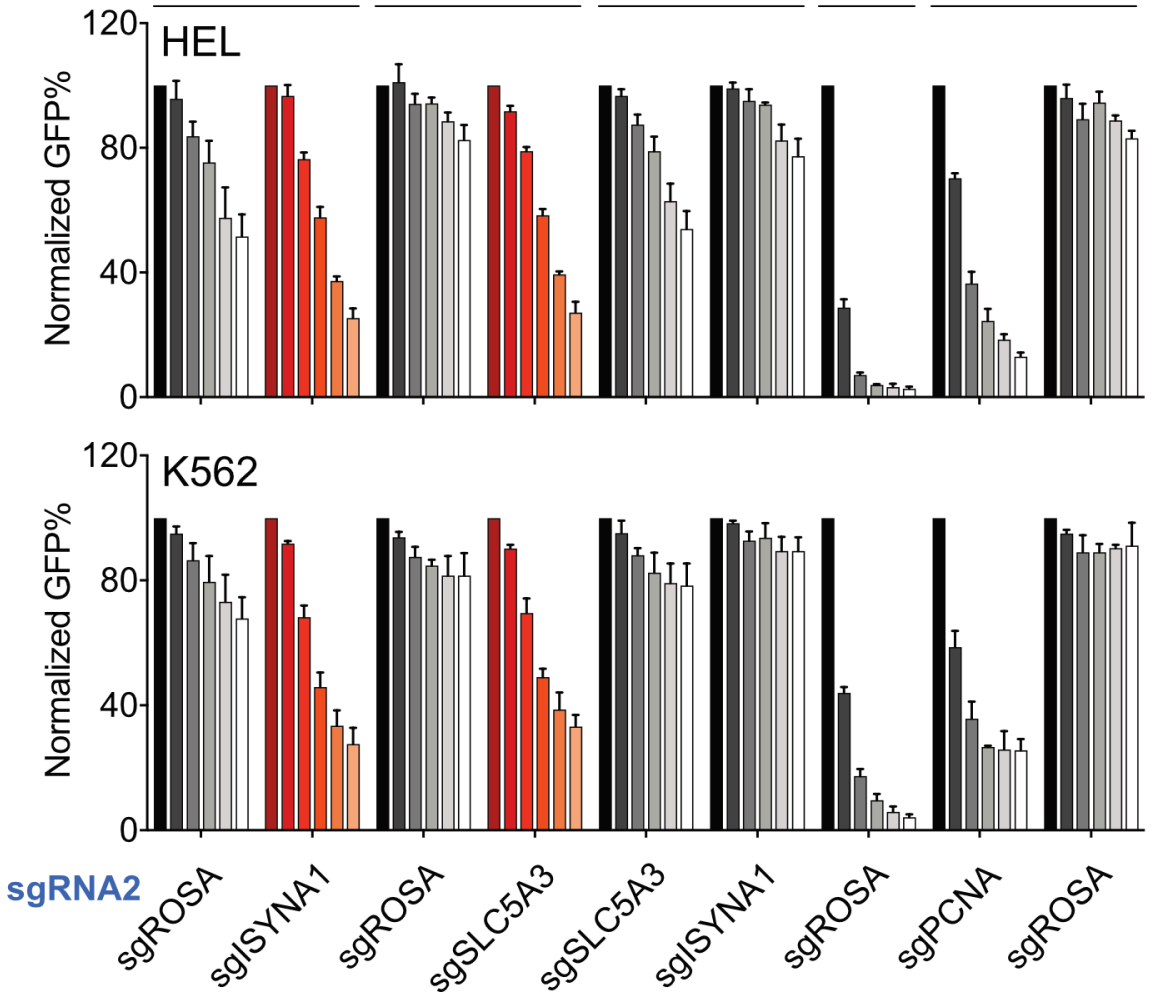

G
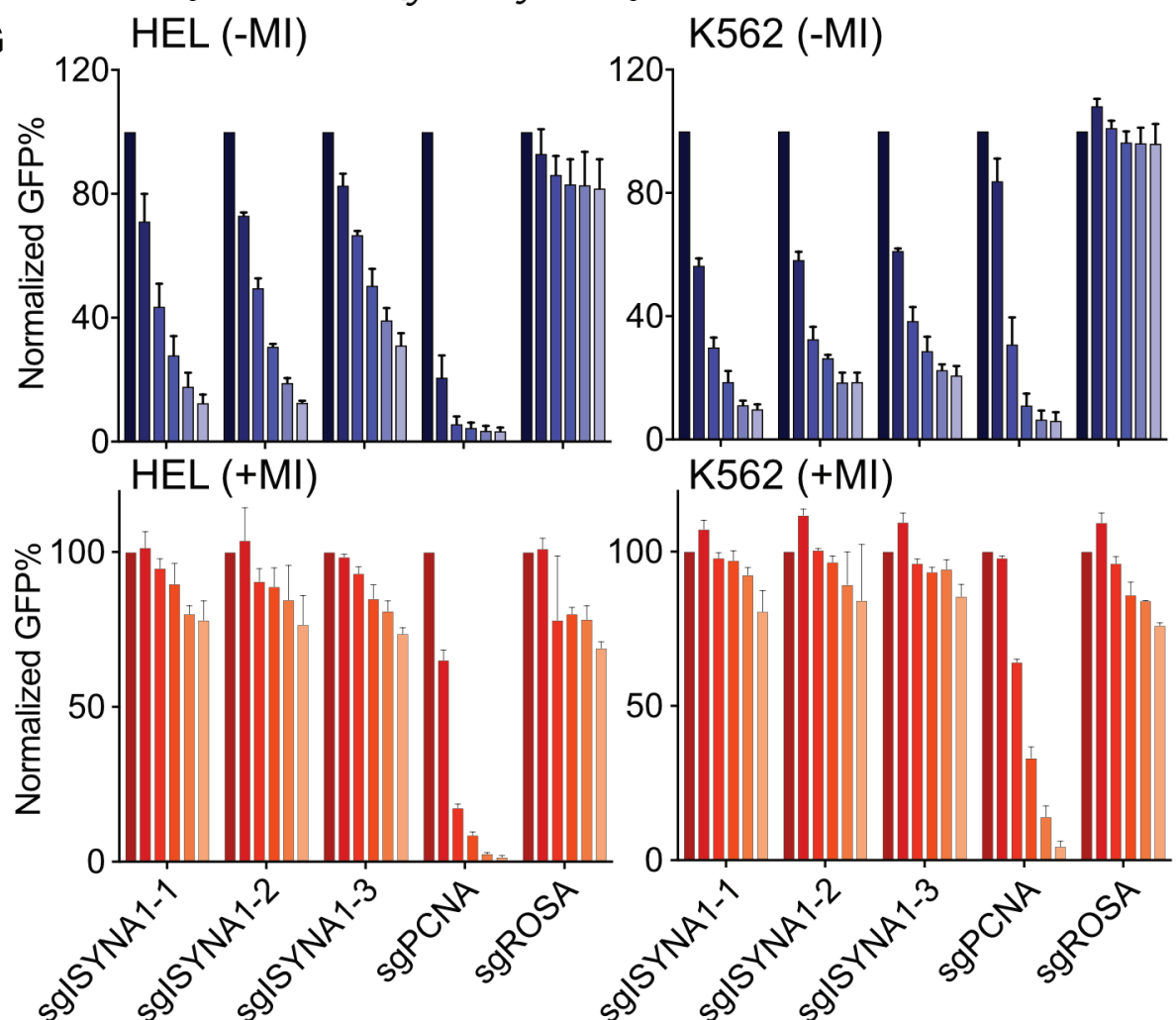
A $\quad$ Author Manuscript Published OnBeFirst on September 16, 2021; DOI: 10.1158/2159-8290.CD-20-1849

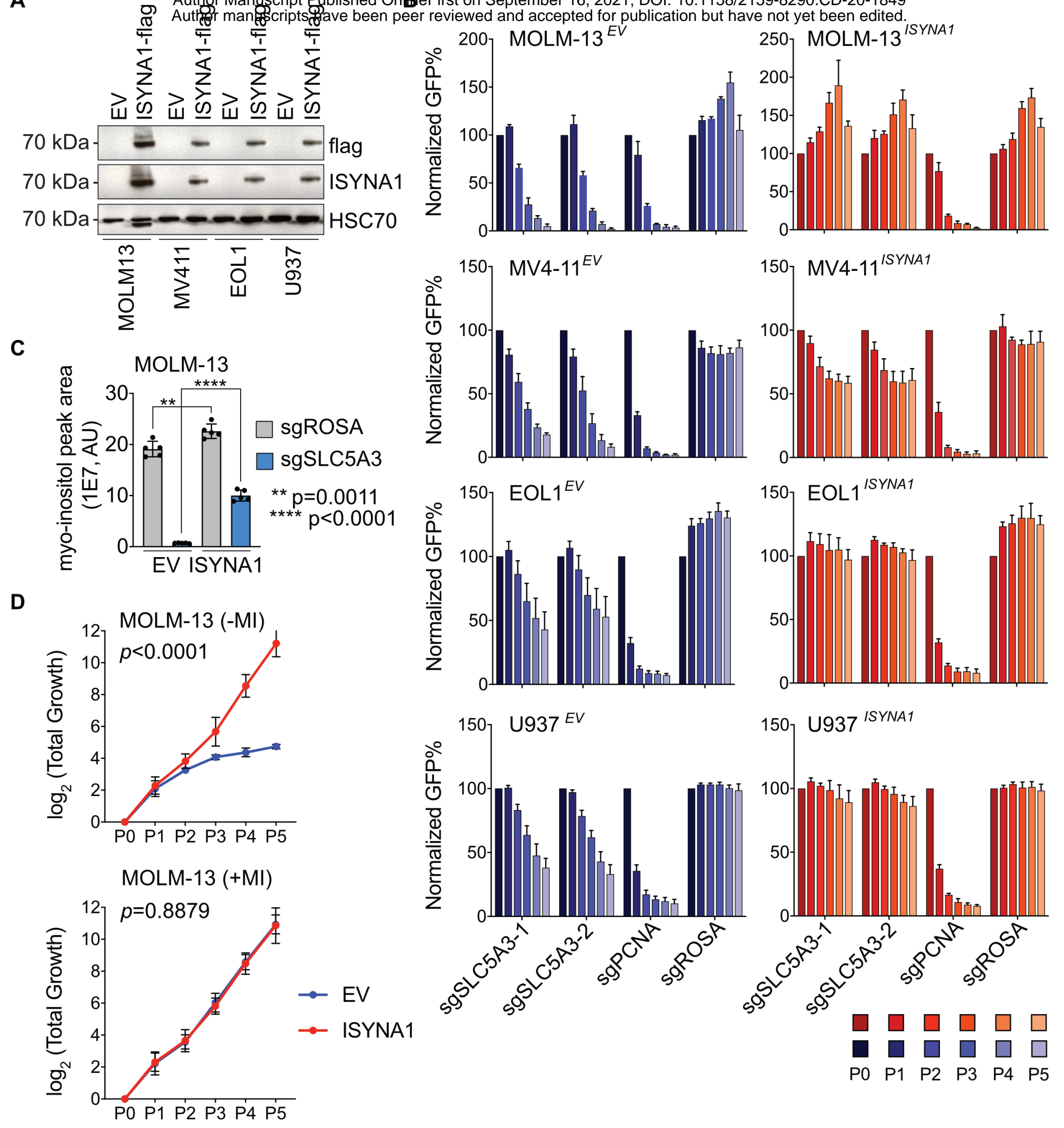




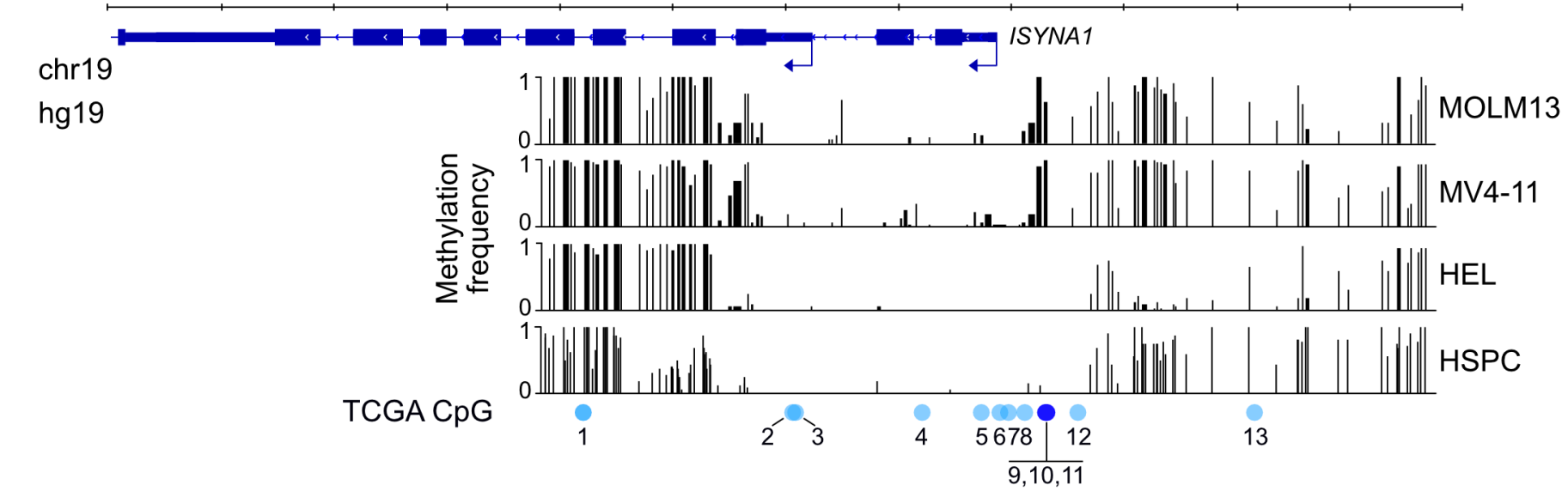

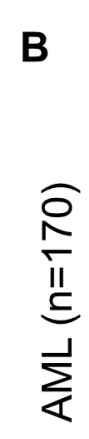

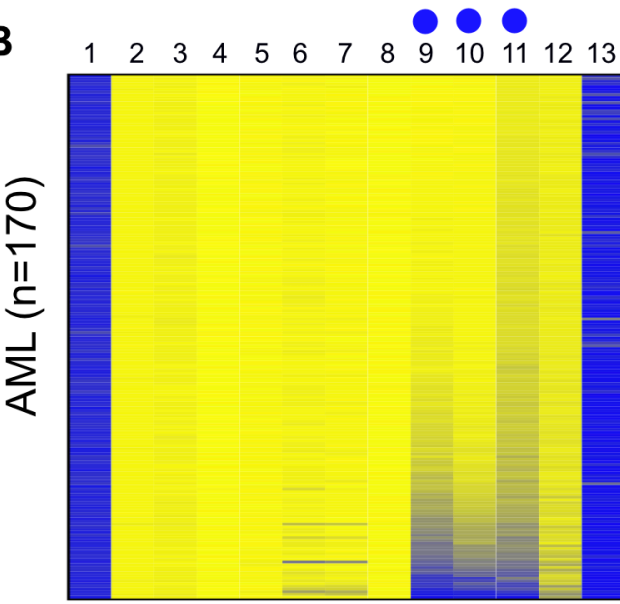

TCGA PanCancer

DNA Methylation

$\begin{array}{llllll}0 & 0.2 & 0.4 & 0.6 & 0.8 & 1.0\end{array}$

E

TCGA PanCancer - AML

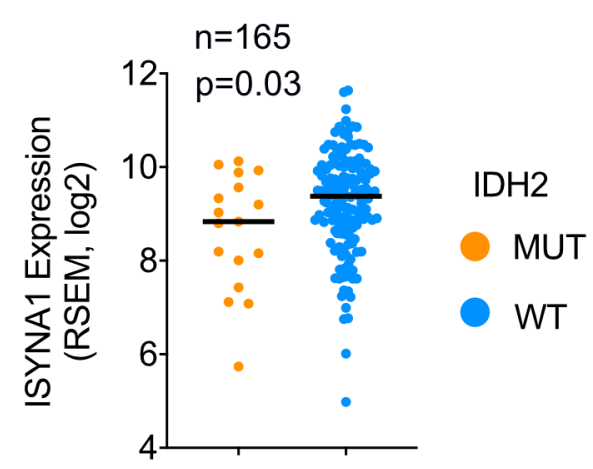

D

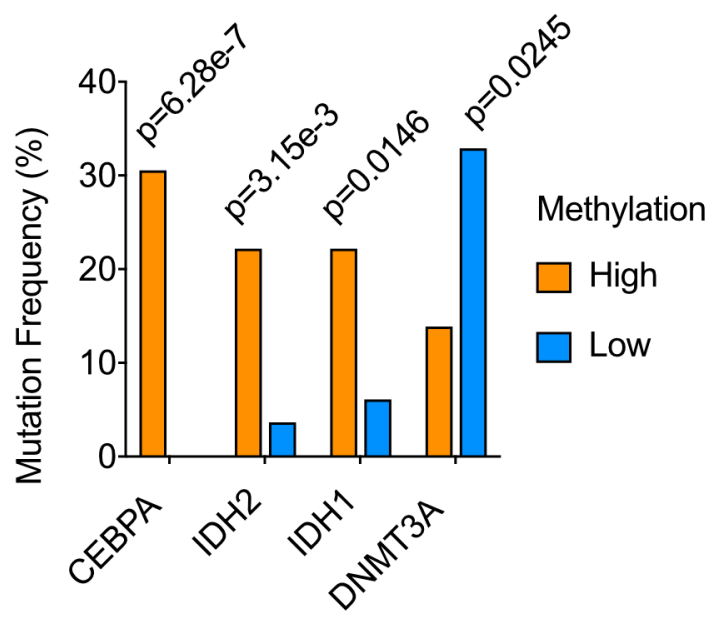

SLC5A3 dependent
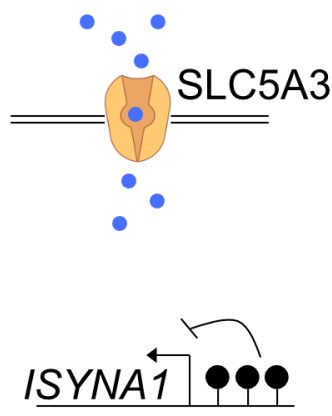

MUT $\square$ WT

\section{F}
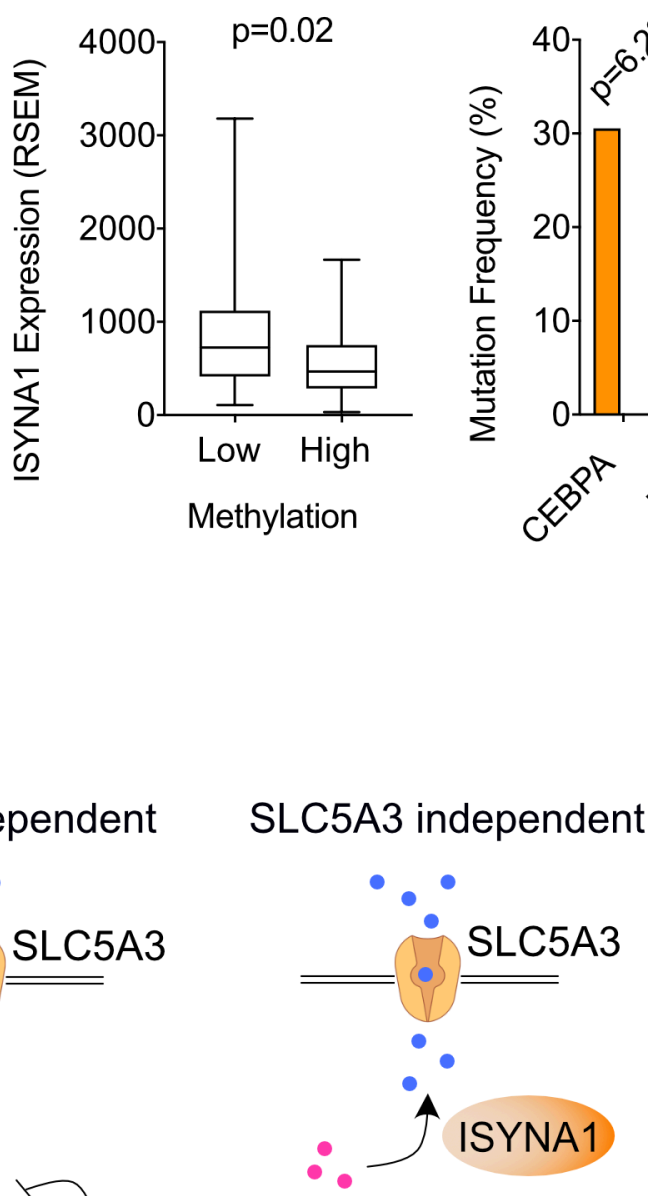

ISYNA 1 OOO
- methylated CpG

$P$ unmethylated $\mathrm{CpG}$

- myo-inositol

- glucose 


\section{CANCER DISCOVERY

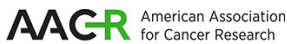

\section{SLC5A3-dependent myo-inositol auxotrophy in acute myeloid leukemia}

Yiliang Wei, Yu-Han Huang, Damianos S Skopelitis, et al.

Cancer Discov Published OnlineFirst September 16, 2021.

Updated version Access the most recent version of this article at: doi:10.1158/2159-8290.CD-20-1849

Supplementary Access the most recent supplemental material at:

Material http://cancerdiscovery.aacrjournals.org/content/suppl/2021/09/16/2159-8290.CD-20-1849.DC1

Author Author manuscripts have been peer reviewed and accepted for publication but have not yet been Manuscript edited.

\section{E-mail alerts Sign up to receive free email-alerts related to this article or journal.}

Reprints and To order reprints of this article or to subscribe to the journal, contact the AACR Publications Subscriptions Department at pubs@aacr.org.

Permissions To request permission to re-use all or part of this article, use this link http://cancerdiscovery.aacrjournals.org/content/early/2021/09/15/2159-8290.CD-20-1849. Click on "Request Permissions" which will take you to the Copyright Clearance Center's (CCC) Rightslink site. 